\title{
Power moments and value distribution of functions
}

\section{Article}

Accepted Version

Hilberdink, T. (2019) Power moments and value distribution of functions. Transactions of the American Mathematical Society, 371. pp. 1-31. ISSN 1088-6850 doi: https://doi.org/10.1090/tran/7581 Available at https://centaur.reading.ac.uk/76912/

It is advisable to refer to the publisher's version if you intend to cite from the work. See Guidance on citing.

To link to this article DOI: http://dx.doi.org/10.1090/tran/7581

Publisher: American Mathematical Society

All outputs in CentAUR are protected by Intellectual Property Rights law, including copyright law. Copyright and IPR is retained by the creators or other copyright holders. Terms and conditions for use of this material are defined in the End User Agreement.

\section{www.reading.ac.uk/centaur}

\section{CentAUR}

Central Archive at the University of Reading

Reading's research outputs online 


\title{
Power moments and value distribution of functions ${ }^{1}$
}

\author{
Titus Hilberdink \\ Department of Mathematics, University of Reading, Whiteknights, \\ PO Box 220, Reading RG6 6AX, UK; t.w.hilberdink@reading.ac.uk
}

\begin{abstract}
In this paper we study various "abscissae" which one can associate to a given function $f$, or rather to the power moments of $f$. These are motivated by long standing open problems in analytic number theory. We show how these abscissae connect to the distribution of values of $f$ in a very elegant way using convex conjugates. This connection allows us to show which abscissae are realizable for both general and more specific arithmetical functions. Further it may give a new approach to, for example, Dirichlet's divisor problem.
\end{abstract}

2010 AMS Mathematics Subject Classification: 11N64, 52A41

Keywords and phrases: power moments of functions, Dirichlet Divisor problem, Lindelöf Hypothesis, convex conjugate, Lindelőf function.

\section{Introduction}

A number of major open problems in analytic number theory show similar characteristics. As typical examples, consider two of these - the Dirichlet divisor problem (DDP) and the Lindelöf Hypothesis (LH). For the DDP, the problem consists of finding the maximal behaviour of the function ${ }^{2}$

$$
\Delta(x)=\sum_{n \leq x}(d(n)-\log n-2 \gamma)
$$

where $d(n)$ is the number of divisors of $n$ and $\gamma$ is Euler's constant. Dirichlet proved in an elementary way that $\Delta(x)=O(\sqrt{x})$, which has been sharpened to $O\left(x^{\frac{1}{3}}\right)$ by Voronoi and subsequently by many researchers, the most recent being Huxley's $O\left(x^{\frac{131}{416}+\varepsilon}\right)(\forall \varepsilon>0)$ (see [13]). On the other hand, Hardy showed it is not $o\left(x^{\frac{1}{4}}\right)$. The problem is to find the optimal exponent which is widely believed to be $\frac{1}{4}$; i.e. $\Delta(x)=O\left(x^{\frac{1}{4}+\varepsilon}\right)$ for all $\varepsilon>0$. Although this has never been proven, it is true in a mean-square average sense as Cramér [5] proved that $\int_{0}^{x} \Delta^{2} \sim c x^{3 / 2}$ for some $c>0$.

Turning to the second problem, $\mathrm{LH}$ is the statement that

$$
\zeta\left(\frac{1}{2}+i t\right)=O\left(t^{\varepsilon}\right)
$$

for all $\varepsilon>0$, where $\zeta(s)$ is Riemann's zeta function and its analytic continuation. It was shown by Hardy and Littlewood that $\zeta\left(\frac{1}{2}+i t\right)=O\left(t^{\frac{1}{6}}\right)$, a bound which has subsequently been improved by many authors, the most recent being Bourgain's $O\left(t^{\frac{53}{342}+\varepsilon}\right)(\forall \varepsilon>0)$ (see [4]). As for $\Delta$, the conjecture is true in a mean-square average sense, for it is known that $\int_{0}^{T}\left|\zeta\left(\frac{1}{2}+i t\right)\right|^{2} d t \sim T \log T$. Furthermore, it is also widely believed as LH is implied by the Riemann Hypothesis $(\mathrm{RH})$.

In both cases, we see that we have a function $f:(0, \infty) \rightarrow \mathbb{C}$ satisfying $f(x)=O\left(x^{\lambda}\right)$ and we need to find the optimal $\lambda$ for which this holds. We have some further information about the mean-square of the function $\int_{0}^{x}|f|^{2}$ and, in these two instances, also about some higher powers. In the first case, the function has an arithmetical character, namely $f(x)=f([x])$, while in the second case, $f$ is holomorphic.

This motivates the following definition. For a function $f:(0, \infty) \rightarrow \mathbb{C}$ on the positive reals which satisfies $f(x)=O\left(x^{\lambda}\right)$ for $x \geq 1$, define the following abscissae: let $\theta$ be the infimum of such $\lambda$, and for

\footnotetext{
${ }^{1}$ To appear in Transactions of the American Mathematical Society.

${ }^{2}$ Usually this is defined by $\Delta(x)=\sum_{n \leq x} d(n)-(x \log x+(2 \gamma-1) x)$, but this differs from our definition by an insignificant $O(\log x)$.
} 
each $p>0$ let

$$
\theta_{p}=\inf \left\{\lambda:\left(\frac{1}{x} \int_{x}^{2 x}|f|^{p}\right)^{1 / p}=O\left(x^{\lambda}\right)\right\}
$$

Of course, the main motivation for studying these 'abscissae' comes from special functions in number theory, but our set up here is very general. When $f$ is 'arithmetical' (i.e. $f(x)=f([x])$ ), then it is a step function of the form

$$
f(x)=\sum_{n \leq x} a_{n}
$$

for some $a_{n}$ and $\theta$ corresponds to the usual abscissa of convergence of the Dirichlet series $\sum a_{n} n^{-s}$ (if $\sum a_{n}$ diverges). Determining these abscissae can be very difficult for 'naturally occurring' examples like $a_{n}=d(n)-\log n-2 \gamma$. For this example it is known that $\theta_{p}=\frac{1}{4}$ for $p \leq 9$ (see [11]) while $\frac{1}{4} \leq \theta \leq \frac{131}{416}$ as mentioned before.

It is straightforward to show that $\theta_{p}$ is increasing as a function of $p$, and further that $p \theta_{p}$ is convex. Natural questions that now arise are (i) when does $\theta_{p} \rightarrow \theta$ as $p \rightarrow \infty$ ? (ii) when do we have $\theta_{p} \equiv \theta$ ?, (iii) what type of functions can $\theta_{p}$ be?

We shall answer these in a very general way by linking these abscissae to a measurement of how often $|f(x)|$ is larger than a given power of $x$; namely by using the sets

$$
U_{\lambda}(X):=\left\{x \in[X, 2 X]:|f(x)| \geq x^{\lambda}\right\} .
$$

Indeed, let $\sigma(\lambda)$ be defined by $\left|U_{\lambda}(X)\right| \ll X^{1-\sigma(\lambda)+\varepsilon}$ for all $\varepsilon>0$ but no $\varepsilon<0$. Then we shall find that $p \theta_{p}=\sigma^{*}(p)$ - the convex conjugate or Legendre-Fenchel transform of $\sigma(\lambda)$ (Theorem 2.2). In this way we give necessary and sufficient conditions for which $\theta_{p} \rightarrow \theta$ and $\theta_{p} \equiv \theta$, as well as showing that $\theta_{p}$ can be any increasing function such that $p \theta_{p}$ is convex (Theorem 2.5). By adjusting the proof, we show in $\S 3$ that this remains essentially true among the 'arithmetical' $f$. The method also provides a possible new approach to Dirichlet's divisor problem: if the slope of $\sigma(\lambda)$ at $\theta-$ is finite and $<9$, then $\theta=\frac{1}{4}$.

In $\S 4$, we consider the abscissae $\mu(\sigma)$ and $\mu_{p}(\sigma)$ (or 'Lindelóf' functions) of the associated Mellin transform. The main result here is that, in a quite general way, $\mu_{2}\left(\theta_{2}\right)=\frac{1}{2}$.

In $\S 5$, we explore a number of examples from diverse areas to highlight the different possible behaviours of $\theta_{p}, \sigma(\lambda)$ and $\mu(\sigma)$.

In $\S 6$, we extend the results to the infinite case by considering a class of functions for which $\theta_{p}$ is finite for all $p>0$ but $\theta=\infty$. A typical 'arithmetical' example is $d\left(\left[e^{x-1}\right]\right)$, where $d$ is the divisor function.

Notation. The symbols $o, O, \ll, \Omega$ are have their usual meanings, namely: (i) $f(x)=O(g(x))$ (equivalently $f(x) \ll g(x))$ if $|f(x)| \leq A g(x)$ for some constant $A$ and all $x \geq x_{0}$ (some $x_{0}$ ); (ii) $f(x)=o(g(x)$ ) if $f(x) / g(x) \rightarrow 0$ as $x \rightarrow \infty$; while (iii) $f(x)=\Omega(g(x))$ is its negation; i.e. there exist $x_{n} \rightarrow \infty$ such that $\left|f\left(x_{n}\right)\right| \geq c\left|g\left(x_{n}\right)\right|$ for some $c>0$ and all $n$.

\section{Various abscissae; $\theta, \theta_{p}$ and $\bar{\theta}$}

Let $S$ denote the class of functions $f:[A, \infty) \rightarrow \mathbb{C}$ (some constant $A>0$ ) which are locally of bounded variation and satisfy $f(x)=O\left(x^{\lambda}\right)$ for some $\lambda$.

Definition 1.1 Let $f \in S$ and let $p>0$. Define $\theta_{p}$ and $\theta$ as follows:

$$
\begin{aligned}
\theta_{p} & =\inf \left\{\lambda:\left(\frac{1}{x} \int_{x}^{2 x}|f|^{p}\right)^{1 / p}=O\left(x^{\lambda}\right)\right\} \\
\theta & =\inf \left\{\lambda: f(x)=O\left(x^{\lambda}\right)\right\} .
\end{aligned}
$$

We note that $\theta_{p}$ can be characterised in terms of $\int_{0}^{x}|f|^{p}$ for large $x$ as follows:

$$
\theta_{p}=\left\{\begin{array}{cl}
\inf \left\{\lambda:\left(\frac{1}{x} \int_{0}^{x}|f|^{p}\right)^{1 / p}=O\left(x^{\lambda}\right)\right\} & \text { if } \int_{0}^{\infty}|f|^{p}=\infty \\
\inf \left\{\lambda:\left(\frac{1}{x} \int_{x}^{\infty}|f|^{p}\right)^{1 / p}=O\left(x^{\lambda}\right)\right\} & \text { if } \int_{0}^{\infty}|f|^{p}<\infty
\end{array} .\right.
$$


Remark 1.1 It is quite possible that either infimum does not exist, for example if $f(x)$ is exponentially small. In this case we write $\theta_{p}=-\infty$ or $\theta=-\infty$. It is also possible to have $\theta_{p}=-\infty$ for all $p$ but $\theta$ finite. For example, if $f(n)=1$ for $n \in \mathbb{N}$ and zero otherwise.

\section{Proposition 1.1 (Basic Properties)}

(a) $\theta_{p}$ increases with $p$;

(b) $\theta_{p} \leq \theta$ for all $p$;

(c) if $\theta_{q}=-\infty$ for some $q$, then $\theta_{p}=-\infty$ for all $p$;

(d) $p \theta_{p}$ is a convex function of $p$;

Proof. (a) Let $q>p>0$. By Hölder's inequality

$$
\begin{aligned}
\int_{x}^{2 x}|f|^{p} & =\int_{x}^{2 x}\left(|f|^{q}\right)^{p / q} \cdot 1^{1-p / q} \leq\left(\int_{x}^{2 x}|f|^{q}\right)^{p / q}\left(\int_{x}^{2 x} 1\right)^{1-p / q} \\
& \ll x^{\left(1+q \theta_{q}+\varepsilon\right) \frac{p}{q}} \cdot x^{1-\frac{p}{q}} \leq x^{1+p \theta_{q}+\varepsilon}
\end{aligned}
$$

and $\theta_{p} \leq \theta_{q}$ follows.

(b) We have $f(x) \ll x^{\theta+\varepsilon}$ for every $\varepsilon>0$, so $\int_{x}^{2 x}|f|^{p} \ll x^{1+p(\theta+\varepsilon)}$. Thus $\theta_{p} \leq \theta$.

(c) Suppose $\theta_{q}=-\infty$. If $p<q$, then (1.2) tells us that $\int_{x}^{2 x}|f|^{p} \ll x^{-A}$ for all $A$, and so $\theta_{p}=-\infty$.

Now suppose $p>q$. Since $|f(x)| \leq x^{c}$ for some $c$, we have

$$
\int_{x}^{2 x}|f|^{p}=\int_{x}^{2 x}|f|^{q}|f|^{p-q} \ll x^{c} \int_{x}^{2 x}|f|^{q} \ll x^{-A}
$$

for all $A$. Thus, again, $\theta_{p}=-\infty$.

(d) Let $p<q<r$. Then by Hölder's inequality

$$
\int_{x}^{2 x}|f|^{q}=\int_{x}^{2 x}|f|^{p\left(\frac{r-q}{r-p}\right)}|f|^{r\left(\frac{q-p}{r-p}\right)} \leq\left(\int_{x}^{2 x}|f|^{p}\right)^{\frac{r-q}{r-p}}\left(\int_{x}^{2 x}|f|^{r}\right)^{\frac{q-p}{r-p}}
$$

Thus

$$
\frac{1}{x} \int_{x}^{2 x}|f|^{q} \leq\left(\frac{1}{x} \int_{x}^{2 x}|f|^{p}\right)^{\frac{r-q}{r-p}}\left(\frac{1}{x} \int_{x}^{2 x}|f|^{r}\right)^{\frac{q-p}{r-p}}=O\left(x^{\frac{r-q}{r-p} p \theta_{p}+\frac{q-p}{r-p} r \theta_{r}+\varepsilon}\right)
$$

for all $\varepsilon>0$. The LHS integral above is $\Omega\left(x^{1+q \theta_{q}-\varepsilon}\right)$, so

$$
q \theta_{q} \leq \frac{r-q}{r-p} p \theta_{p}+\frac{q-p}{r-p} r \theta_{r}, \quad \text { as required. }
$$

\section{Remarks 1.2}

(a) As a consequence of convexity, we find that (i) $\theta_{p}$ is continuous, and (ii) $\theta_{p_{0}}=\theta$ for some $p_{0}$ implies $\theta_{p} \equiv \theta$ (also using monotonicity). Furthermore, $p\left(\theta-\theta_{p}\right)$ is increasing. For it is concave, and so for $p<q<r$,

$$
(r-p) q\left(\theta-\theta_{q}\right) \geq(q-p) r\left(\theta-\theta_{r}\right)+(r-q) p\left(\theta-\theta_{p}\right) \geq(r-q) p\left(\theta-\theta_{p}\right),
$$

which implies $r\left(p\left(\theta-\theta_{p}\right)-q\left(\theta-\theta_{q}\right)\right) \leq p q\left(\theta_{q}-\theta_{p}\right)$. If $p\left(\theta-\theta_{p}\right)>q\left(\theta-\theta_{q}\right)$ then the LHS can be made arbitrarily large by increasing $r$ - a contradiction.

Let $\Delta=\lim _{p \rightarrow \infty} p\left(\theta-\theta_{p}\right) \in[0, \infty]$. Thus $\Delta=0$ if and only if $\theta_{p} \equiv \theta$. 
(b) Using $\theta_{r} \leq \theta$, letting $r \rightarrow \infty$ in (1.3), and putting $p=1$ gives, for $q>1$

$$
q \theta_{q} \leq \theta_{1}+(q-1) \theta
$$

So, for example, $2 \theta_{2} \leq \theta_{1}+\theta$.

The abscissa $\bar{\theta}$. For $f \in S$, let $V_{f}(x)$ denote the total variation of $f$ on $[0, x]$. Let $\bar{\theta}$ be defined by

$$
\bar{\theta}=\inf \left\{\lambda: V_{f}(x)=O\left(x^{\lambda}\right)\right\} .
$$

It may happen that there is no $\lambda$ for which $V_{f}(x)=O\left(x^{\lambda}\right)$; in this case we say $\bar{\theta}=\infty$. Trivially, $\bar{\theta} \geq \theta$.

\section{Basic facts}

(a) If $f(x)=\sum_{n \leq x} a_{n}$, then $V_{f}(x)=\sum_{n \leq x}\left|a_{n}\right|$ and $\bar{\theta}$ corresponds to the abscissae of absolute convergence of $\sum a_{n} n^{-s}$ (if $\sum\left|a_{n}\right|$ diverges). Note that in this case $\bar{\theta} \leq \theta+1$ (if $\theta \geq-1$ ). For

$$
V_{f}(x)=\sum_{n \leq x}|f(n)-f(n-1)| \leq 2 \sum_{n \leq x}|f(n)| \ll \sum_{n \leq x} n^{\theta+\varepsilon} \ll x^{\theta+1+\varepsilon} .
$$

(b) If $f$ is absolutely continuous, then $V_{f}(x)=\int_{0}^{x}\left|f^{\prime}\right|$ (see [28], p.393).

Unlike in case (a), in general we have no control over the size of $\bar{\theta}-\theta$. For example, let $f(x)=\sin \left(x^{\lambda}\right)$ where $\lambda>0$. Then $\theta=0$ while $\bar{\theta}=\lambda$, since

$$
V_{f}(x)=\int_{0}^{x}\left|\lambda t^{\lambda-1} \cos \left(t^{\lambda}\right)\right| d t \asymp x^{\lambda} .
$$

In fact it may happen that $\bar{\theta}=\infty$ (see example (iv) in section 3).

(c) Observe that $\theta$ and $\theta_{p}$ depend only of $|f|$ (i.e. $f$ and $|f|$ have the same abscissae) while $\bar{\theta}$ may be different for $f$ and $|f|$.

\section{Connecting $\theta_{p}$ to the distribution of $f$; the function $\sigma(\lambda)$}

In order to answer the questions about the possibilities for $\theta_{p}$, we define the following quantities which measure how often $f$ is 'large'.

Definition 2.1 Let $f \in S$ with finite abscissa $\theta$. For $\lambda \in \mathbb{R}$ and $x \geq 1$ let

$$
U_{\lambda}(x)=\left\{x \leq t \leq 2 x:|f(t)| \geq t^{\lambda}\right\},
$$

and denote the Lebesque measure of $U_{\lambda}(x)$ by $\left|U_{\lambda}(x)\right|$.

Note that for $\lambda<\mu$ we have $U_{\lambda}(x) \supset U_{\mu}(x),\left|U_{\lambda}(x)\right| \leq x$, while $U_{\lambda}(x)=\emptyset$ for $\lambda>\theta$ and $x$ sufficiently large. As $\left|U_{\lambda}(\cdot)\right| \in S$, we may define the function $\sigma(\lambda) \in[0, \infty]$ via

$$
\left|U_{\lambda}(x)\right|=O\left(x^{1-\sigma(\lambda)+\varepsilon}\right) \quad \text { for all } \varepsilon>0 \text { but no } \varepsilon<0 .
$$

We see immediately that (i) $\sigma(\lambda)$ is increasing, (ii) $\sigma(\lambda) \geq 0$ for all $\lambda$, and (iii) $\sigma(\lambda)=\infty$ for $\lambda>\theta$.

\subsection{Connecting $\theta_{p}$ and $\sigma(\lambda)$}

Given the example from Remark 1.1, it is clear that we need some sort of regularity condition on $f$ in order for $\theta_{p} \rightarrow \theta$ as $p \rightarrow \infty$ to hold. In Theorem 2.1 below, we find a necessary and sufficient condition for $\theta_{p} \rightarrow \theta$, as well as for $\theta_{p} \equiv \theta$ using $\sigma(\lambda)$.

In Theorem 2.2, we go further and show how $\sigma(\lambda)$ determines $\theta_{p}$. 


\section{Theorem 2.1}

Let $f \in S$ with abscissae $\theta$ and $\theta_{p}$. Then

$$
\begin{aligned}
\text { (i) }) \quad \theta_{p} \rightarrow \theta & \Longleftrightarrow \sigma(\lambda)<\infty \quad \text { for all } \lambda<\theta \\
\text { (ii }) \quad \theta_{p} \equiv \theta & \Longleftrightarrow \sigma(\lambda)=0 \quad \text { for all } \lambda<\theta .
\end{aligned}
$$

Proof. (i) $(\Leftarrow)$ Let $\varepsilon>0$. By assumption, there exists $a$ such that $\left|U_{\theta-\varepsilon}(x)\right|=\Omega\left(x^{-a}\right)$. Hence

$$
x^{1+p \theta_{p}+\varepsilon} \gg \int_{x}^{2 x}|f|^{p} \geq \int_{U_{\theta-\varepsilon}(x)} t^{p(\theta-\varepsilon)} d t \asymp x^{p(\theta-\varepsilon)}\left|U_{\theta-\varepsilon}(x)\right|=\Omega\left(x^{p(\theta-\varepsilon)-a}\right) .
$$

Hence $1+p \theta_{p}+\varepsilon \geq p(\theta-\varepsilon)-a$; i.e.

$$
\theta_{p} \geq \theta-\varepsilon-\frac{1+a+\varepsilon}{p}
$$

As such, $\lim \inf _{p \rightarrow \infty} \theta_{p} \geq \theta-\varepsilon$. But this holds for every $\varepsilon>0$, so $\liminf _{p \rightarrow \infty} \theta_{p} \geq \theta$, and $\theta_{p} \rightarrow \theta$ follows.

$(\Rightarrow)$ Suppose now that $\sigma(\lambda)=\infty$ for some $\lambda<\theta$; i.e. $\left|U_{\lambda}(x)\right|=O\left(x^{-A}\right)$ for all $A$. Then, for all $\varepsilon>0$, we have

$$
\int_{x}^{2 x}|f|^{p} \ll \int_{U_{\lambda}(x)} t^{p(\theta+\varepsilon)} d t+\int_{[x, 2 x] \backslash U_{\lambda}(x)} t^{p \lambda} d t \ll x^{p(\theta+\varepsilon)}\left|U_{\lambda}(x)\right|+x^{1+p \lambda} \ll x^{1+p \lambda}
$$

for every $\varepsilon>0$. The LHS is $\Omega\left(x^{1+p \theta_{p}-\varepsilon}\right)$, so $\theta_{p} \leq \lambda<\theta$. Hence $\theta_{p} \not \rightarrow \theta$. Now

(ii) $(\Rightarrow)$ Suppose $\theta_{p} \equiv \theta$ but that $\sigma(\lambda)>0$ for some $\lambda<\theta$. Then $\left|U_{\lambda}(x)\right|=O\left(x^{1-\delta}\right)$ for some $\delta>0$.

$$
\int_{x}^{2 x}|f|^{p}=\int_{U_{\lambda}(x)}|f|^{p}+\int_{[x, 2 x] \backslash U_{\lambda}(x)}|f|^{p} \ll x^{p(\theta+\varepsilon)}\left|U_{\lambda}(x)\right|+x^{1+p \lambda} \ll x^{1+p(\theta+\varepsilon)-\delta}+x^{1+p \lambda}
$$

using the fact that for $t \in[x, 2 x] \backslash S_{\lambda}(x),|f(t)| \leq t^{\lambda} \ll x^{\lambda}$. The LHS above is $\Omega\left(x^{1+p\left(\theta_{p}-\varepsilon\right)}\right)$, so

$$
\theta_{p} \leq \max \left\{\theta-\frac{\delta}{p}, \lambda\right\}<\theta
$$

- a contradiction.

$(\Leftarrow)$ For the converse implication, suppose that $\sigma(\lambda)=0$ for all $\lambda<\theta$. Thus $\left|U_{\lambda}(x)\right|=\Omega\left(x^{1-\varepsilon}\right)$ for all $\varepsilon>0$ and $\lambda<\theta$. Hence

$$
\int_{x}^{2 x}|f|^{p} \geq \int_{U_{\lambda}(x)}|f|^{p} \gg x^{p \lambda}\left|U_{\lambda}(x)\right|=\Omega\left(x^{1+p \lambda-\varepsilon}\right) .
$$

Thus $\theta_{p} \geq \lambda$ for every $\lambda<\theta$. It follows that $\theta_{p} \geq \theta$, and hence equality must occur.

\section{Remarks 2.1}

(i) For Theorem 2.1(i), we could equally well work with smaller intervals. For example, put $T_{\lambda}(x)=$ $\left\{x \leq t \leq x+1:|f(t)| \geq t^{\lambda}\right\}$ with $\left|T_{\lambda}(x)\right|$ its Lebesque measure. Then $\theta_{p} \rightarrow \theta$ if and only if the same condition holds with $U$ replaced by $T$. This follows immediately from the facts that $\left|T_{\lambda}(x)\right| \leq\left|U_{\lambda}(x)\right|$ and $\left|U_{\lambda}(x)\right| \leq \sum_{0 \leq k \leq x}\left|T_{\lambda}(x+k)\right|$.

(ii) If in Theorem 2.1(i) we assume that the condition holds uniformly; i.e. there exists $a(>-1)$ such that $\forall \varepsilon>0,\left|U_{\theta-\varepsilon}(x)\right|=\Omega\left(x^{-a}\right)$, then the same argument as in the $(\Leftarrow)$ direction of the above proof shows that we have the stronger lower bound

$$
\theta_{p} \geq \theta-\frac{1+a}{p}
$$

i.e. $p\left(\theta-\theta_{p}\right)$ remains bounded and so also $\Delta \leq a+1$. 
(iii) In the special case that $f$ is a step function of the form $\sum_{n \leq x} a_{n}$, then the condition holds uniformly with $a=0$. For, given $\varepsilon>0$, there exists a sequence of positive integers $n_{k} \nearrow \infty$ for which $\left|f\left(n_{k}\right)\right| \geq$ $\left(n_{k}+1\right)^{\theta-\varepsilon}$. Hence for $x \in\left[n_{k}, n_{k}+1\right],|f(x)|=\left|f\left(x_{n}\right)\right| \geq x^{\theta-\varepsilon}$ and $\left|U_{\theta-\varepsilon}\left(n_{k}\right)\right| \geq\left|T_{\theta-\varepsilon}\left(n_{k}\right)\right|=1$. Thus $\theta_{p} \geq \theta-\frac{1}{p}$.

\section{Theorem 2.2}

Let $f \in S$ for which $\theta_{p} \rightarrow \theta$ as $p \rightarrow \infty$. Then

$$
p \theta_{p}=\sigma^{*}(p):=\sup _{\lambda \in \mathbb{R}}(p \lambda-\sigma(\lambda)) .
$$

Proof. We first prove $p \theta_{p} \geq \sigma^{*}(p)$.

Let $\varepsilon>0$. By definition of $\sigma^{*}(p)$, there exists $\lambda$ such that $p \lambda-\sigma(\lambda)>\sigma^{*}(p)-\varepsilon$. Hence

$$
\begin{aligned}
x^{1+p \theta_{p}+\varepsilon} & \gg \int_{x}^{2 x}|f|^{p} \geq \int_{U_{\lambda}(x)} t^{p \lambda} d t \gg x^{p \lambda}\left|U_{\lambda}(x)\right| \\
& =\Omega\left(x^{p \lambda+1-\sigma(\lambda)-\varepsilon}\right)=\Omega\left(x^{1+\sigma^{*}(p)-2 \varepsilon}\right) .
\end{aligned}
$$

This holds for all $\varepsilon>0$, so $p \theta_{p} \geq \sigma^{*}(p)$.

Now we show the reverse inequality. Let $\lambda$ be sufficiently small so that

$$
\int_{[x, 2 x] \backslash U_{\lambda}(x)}|f|^{p} \ll x^{c}
$$

for some $c<1+p \theta_{p}$. This is possible since the integral is $\ll \int_{x}^{2 x} t^{p \lambda} d t \ll x^{1+p \lambda}$, so it suffices to have $\lambda<\theta_{p}$. As such, $\int_{U_{\lambda}(x)}|f|^{p}=\int_{x}^{2 x}|f|^{p}+O\left(x^{c}\right)$. Now let $K \in \mathbb{N}$ (large). Choose $\mu>\theta$ and let $x$ be sufficiently large so that $U_{\mu}(x)=\emptyset$. Then

$$
\sum_{k=1}^{K} \int_{U_{\frac{(k-1) \mu}{K}+\lambda}(x) \backslash U_{\frac{k \mu}{K}+\lambda}(x)}|f|^{p}=\int_{U_{\lambda}(x)}|f|^{p}-\int_{U_{\mu}(x)}|f|^{p}=\int_{U_{\lambda}(x)}|f|^{p} .
$$

The $k^{\text {th }}$-term on the left is

$$
\ll x^{p \frac{k \mu}{K}+p \lambda}\left|U_{\frac{(k-1) \mu}{K}+\lambda}(x)\right| \ll x^{\frac{p k \mu}{K}+p \lambda+1-\sigma\left(\frac{(k-1) \mu}{K}+\lambda\right)+\varepsilon} \leq x^{\sigma^{*}(p)+1+\frac{p \mu}{K}+\varepsilon}
$$

for all $\varepsilon>0$, since $t \notin U_{\frac{k \mu}{K}+\lambda}(x) \Rightarrow|f(t)|<t^{\frac{k \mu}{K}+\lambda} \ll x^{\frac{k \mu}{K}+\lambda}$. Thus

$$
\int_{U_{\lambda}(x)}|f|^{p} \ll x^{\sigma^{*}(p)+1+\frac{p \mu}{K}+\varepsilon} .
$$

The LHS is $\int_{x}^{2 x}|f|^{p}+O\left(x^{c}\right)=\Omega\left(x^{1+p \theta_{p}-\varepsilon}\right)$ for $\varepsilon$ sufficiently small (since $c<1+p \theta_{p}$ ). Thus $p \theta_{p} \leq$ $\sigma^{*}(p)+\frac{p \mu}{K}$. But $K$ was arbitrarily chosen. Hence $p \theta_{p} \leq \sigma^{*}(p)$ and the result follows.

\section{Remarks 2.3}

(a) From the theory of convexity, given $g$, the quantity

$$
g^{*}(y)=\sup _{x \in \mathbb{R}}(x y-g(x))
$$

is well-known and has been studied in great detail. It is called the convex conjugate of $g$, or sometimes the Legendre-Fenchel transform of $g$. It is necessarily convex where it is defined (whether 
or not $g$ is convex). Furthermore, $g^{* *}$ is the convex envelope of $g$ - the largest convex function $h$ such that $h \leq g$. In particular, if $g$ is convex, then $g^{* *}=g$ (see [21] for a detailed discussion).

Letting $\tilde{\sigma}$ denote the convex envelope of $\sigma$ (i.e. the largest convex function $h$ such that $h \leq \sigma$ ), we find that

$$
p \theta_{p}=\sup _{\lambda \in \mathbb{R}}(p \lambda-\tilde{\sigma}(\lambda)) .
$$

For $\tilde{\sigma}$ is convex, so $\tilde{\sigma}^{* *}=\tilde{\sigma}=\sigma^{* *}$ (from above). Hence $\tilde{\sigma}^{*}=\sigma^{* * *}=\sigma^{*}$, which is $(2.2)$.

(b) We therefore see that the function $\sigma(\lambda)$ determines $\theta_{p}$ (given $\theta$ ), but in the other direction $\theta_{p}$ only determines $\tilde{\sigma}(\lambda)$ (and not $\sigma(\lambda)$ ).

2.2 Possible $\theta_{p}$ and $\sigma(\lambda)$

The function $\sigma(\lambda)$ gives a very clear picture of the distribution of values of $|f(t)|$. We can use it to show that any $\phi_{p}$ satisfying a), b), and d) of Proposition 1.1 can be realised as some $\theta_{p}$. Indeed we show that, essentially, any non-negative increasing function on $(-\infty, \theta)$ can be realised as a $\sigma$-function. First we need the following Lemma, which shows that in order to calculate $\sigma(\lambda)$ it is enough to know the behaviour of $\left|U_{\lambda}\left(2^{n}\right)\right|$.

\section{Lemma 2.3}

Let $\lambda \in \mathbb{R}$. Suppose there exists $\sigma$ such that $\left|U_{\lambda}\left(2^{n}\right)\right|=O\left(2^{n(1-\sigma+\varepsilon)}\right)$ for every $\varepsilon>0$ but for no $\varepsilon<0$. Then $\sigma=\sigma(\lambda)$.

Proof. Every $x \geq 1$ lies in some interval $\left[2^{n}, 2^{n+1}\right)$. As such $U_{\lambda}(x) \subset U_{\lambda}\left(2^{n}\right) \cup U_{\lambda}\left(2^{n+1}\right)$ and so

$$
\left|U_{\lambda}(x)\right| \leq\left|U_{\lambda}\left(2^{n}\right)\right|+\left|U_{\lambda}\left(2^{n+1}\right)\right| \ll 2^{n(1-\sigma+\varepsilon)} \ll x^{1-\sigma+\varepsilon}
$$

for every $\varepsilon>0$. That $\left|U_{\lambda}(x)\right|=\Omega\left(x^{1-\sigma-\varepsilon}\right)$ follows directly from the $x=2^{n}$ case. Hence $\sigma=\sigma(\lambda)$.

\section{Theorem 2.4}

Let $\phi \in \mathbb{R}$ and let $k:(-\infty, \phi) \rightarrow[0, \infty)$ be increasing and left-continuous. Then there exists $f \in S$ for which $\theta=\phi$ and $\sigma(\lambda)=k(\lambda)$ for $\lambda<\theta$.

Proof. Let $k^{\leftarrow}$ denote the generalised inverse ${ }^{3}$ of $k$. Let $f(x)=x^{\tau(x)}$ for $x \geq 1$ and zero otherwise, where $\tau$ is defined on intervals $\left(2^{n}, 2^{n+1}\right]\left(n \in \mathbb{N}_{0}\right)$ by

$$
\tau\left(2^{n}+2^{t n}\right)=k^{\leftarrow}(1-t)=\sup \{y<\phi: k(y) \leq 1-t\} .
$$

Note that $\tau(x) \leq \phi$ always with equality when $x=2^{n}+$ (i.e. $t \rightarrow-\infty$ above). Thus we see that $\theta=\phi$. We make the following convention: if there is no $y<\theta$ such that $k(y) \leq 1-t$ (e.g. when $k$ is bounded below by a positive constant), then we take the sup to be $-\infty$ and $f\left(2^{n}+2^{t n}\right)=0$.

Now consider $U_{\lambda}\left(2^{n}\right)$ for $\lambda<\theta$. We have

$$
U_{\lambda}\left(2^{n}\right)=\left\{x \in\left[2^{n}, 2^{n+1}\right]: \tau(x) \geq \lambda\right\}=\left\{2^{n}+2^{t n}:-\infty \leq t \leq 1, k^{\leftarrow}(1-t) \geq \lambda\right\} .
$$

If $t \leq 1-k(\lambda)$, then $k^{\leftarrow}(1-t) \geq \lambda$, and so $2^{n}+2^{t n} \in U_{\lambda}\left(2^{n}\right)$. Thus $U_{\lambda}\left(2^{n}\right) \supset\left(2^{n}, 2^{n}+2^{n(1-k(\lambda))}\right)$ and $\left|U_{\lambda}\left(2^{n}\right)\right| \geq 2^{n(1-k(\lambda))}$.

On the other hand, if $t>1-k(\lambda-\varepsilon)$ (where $\varepsilon>0$ ), then $k^{\leftarrow}(1-t) \leq \lambda-\varepsilon<\lambda$ and $2^{n}+2^{t n} \notin U_{\lambda}\left(2^{n}\right)$. Thus $U_{\lambda}\left(2^{n}\right) \subset\left[2^{n}, 2^{n}+2^{n(1-k(\lambda-\varepsilon))}\right)$. Thus $2^{n(1-k(\lambda))} \leq\left|U_{\lambda}\left(2^{n}\right)\right| \leq 2^{n(1-k(\lambda-\varepsilon))}$. But $k$ is left-continuous so, letting $\varepsilon \rightarrow 0+$ gives $\left|U_{\lambda}\left(2^{n}\right)\right|=2^{n(1-k(\lambda))}$. By Lemma 2.3 , it follows that $\sigma(\lambda)=k(\lambda)$ for all $\lambda<\theta$.

Remark 2.4 In Theorem 2.4, it is clear that $\bar{\theta}=\theta$. However, since $\theta$ and $\theta_{p}$ only depend on $|f|$, we could, by taking $f_{1}=e^{i \varphi} f$ with $\varphi$ real, find examples where $\bar{\theta}$ is any given real number greater than $\theta$

\footnotetext{
${ }^{3}$ For $f:(-\infty, X) \rightarrow \mathbb{R}$, the generalised inverse is defined to be the function $f^{\leftarrow}(x)=\inf \{y<X: f(y)>x\}$. If $f$ is increasing, we automatically have $f^{\leftarrow}(x)=\sup \{y<X: f(y) \leq x\}$.
} 
(by making $\varphi$ oscillate).

\section{Theorem 2.5}

Let $\phi, \phi_{p} \in \mathbb{R}(p>0)$ such that $\phi_{p} \nearrow \phi$ as $p \rightarrow \infty$ and $p \phi_{p}$ is convex. Then there exists $f \in S$ such that $\theta=\phi$ and $\theta_{p}=\phi_{p}$.

Proof. Consider the conjugate function of $p \phi_{p}$ :

$$
g(\lambda)=\sup _{p>0}\left(p \lambda-p \phi_{p}\right)
$$

By the general theory, $g$ is convex but also we observe that (i) $g(\lambda)=\infty$ for $\lambda>\phi$ while $g(\lambda)<\infty$ for $\lambda<\phi$, (ii) $g \geq 0$ and (iii) $g$ is increasing.

For if $\lambda=\phi+\delta$, with $\delta>0$, then $p\left(\lambda-\phi_{p}\right) \geq p \delta$ and the supremum can be made arbitrarily large. On the other hand, if $\lambda<\phi$ then, with $p$ sufficiently large, $\phi_{p}>\lambda$ and so $p\left(\lambda-\phi_{p}\right)<0$. For (ii), note that $p\left(\lambda-\phi_{p}\right) \geq p(\lambda-\phi)$, which can be made arbitrarily small, and hence $g(\lambda) \geq 0$. (iii) is immediate. Thus

By Theorem 2.4, we can find $f \in S$ for which $\theta=\phi$ and $\sigma \equiv g$. Since $p \phi_{p}$ is convex, $g^{*}(p)=p \phi_{p}$.

$$
p \phi_{p}=\sup _{\lambda<\phi}(p \lambda-g(\lambda))=\sup _{\lambda \in \mathbb{R}}(p \lambda-\sigma(\lambda)) .
$$

The RHS is $p \theta_{p}$ by Theorem 2.2 , and the result follows.

\subsection{The behaviour of $\theta_{p}$ for $p$ large}

The behaviour of $\sigma(\lambda)$ for $\lambda$ near $\theta$ is closely related to the behaviour of $\theta_{p}$ for $p$ large. The function $\sigma(\lambda)$ is increasing, so $\lim _{\lambda \rightarrow \theta-} \sigma(\lambda)$ exists or is $\infty$. Denote this by $\sigma(\theta-)$ in either case. Recall that $\Delta=\lim _{p \rightarrow \infty} p\left(\theta-\theta_{p}\right) \in[0, \infty]$.

\section{Proposition 2.6}

$p\left(\theta-\theta_{p}\right)$ is bounded above if and only if $\sigma(\theta-)<\infty$, in which case $\sigma(\theta-)=\Delta$.

Proof. $(\Leftarrow)$ Suppose $\sigma(\theta-)<\infty$. For every $\lambda<\theta$ and $p>0, p \theta_{p} \geq p \lambda-\sigma(\lambda)$, or $\sigma(\lambda) \geq p\left(\lambda-\theta_{p}\right)$. Letting $\lambda \rightarrow \theta$ gives $\sigma(\theta-) \geq p\left(\theta-\theta_{p}\right)$ for each $p>0$. Thus $\sigma(\theta-) \geq \Delta$.

$(\Rightarrow)$ Suppose $\Delta<\infty$. Then $\sup _{\lambda<\theta}(p \lambda-\sigma(\lambda))=p \theta_{p} \geq p \theta-\Delta$ for all $p>0$. But for every $\delta>0$,

$$
\sup _{\lambda \leq \theta-\frac{\Delta+\delta}{p}}(p \lambda-\sigma(\lambda)) \leq \sup _{\lambda \leq \theta-\frac{\Delta+\delta}{p}} p \lambda=p \theta-\Delta-\delta<p \theta-\Delta .
$$

Thus

$$
p \theta-\Delta \leq \sup _{\theta-\frac{\Delta+\delta}{p}<\lambda<\theta}(p \lambda-\sigma(\lambda)) \leq p \theta-\sigma\left(\theta-\frac{\Delta+\delta}{p}\right) .
$$

Hence $\sigma\left(\theta-\frac{\Delta+\delta}{p}\right) \leq \Delta$ for all $p, \delta>0$; i.e. $\sigma(\theta-) \leq \Delta$.

In many examples (eg. in the 'extremal' case - see section 3.3), we find that $p\left(\theta-\theta_{p}\right)$ is constant from some point onwards. We now give a necessary and sufficient condition for this to hold.

In order to state it we mention some facts concerning the smoothness of convex functions (see [21]). For $g$ convex on an open interval, both left and right derivatives exist; that is

$$
g_{-}^{\prime}(x)=\lim _{h \rightarrow 0-} \frac{g(x+h)-g(x)}{h} \quad \text { and } \quad g_{+}^{\prime}(x)=\lim _{h \rightarrow 0+} \frac{g(x+h)-g(x)}{h}
$$

exist, and both functions are increasing. By convexity it follows that $g_{-}^{\prime}(x) \leq g_{+}^{\prime}(x) \leq g_{-}^{\prime}(y)$ whenever $x<y$. Furthermore,

$$
g(y)-g(x)=\int_{x}^{y} g_{-}^{\prime}=\int_{x}^{y} g_{+}^{\prime} .
$$


In particular, with $\tilde{\sigma}$ denoting the convex envelope of $\sigma$, we have

$$
\tilde{\sigma}_{-}^{\prime}(x) \leq \tilde{\sigma}_{+}^{\prime}(x) \leq \tilde{\sigma}_{-}^{\prime}(y) \leq \tilde{\sigma}_{+}^{\prime}(y) \quad(x<y<\theta) .
$$

Thus both $\tilde{\sigma}_{ \pm}^{\prime}(\theta-)=\lim _{\delta \rightarrow 0+} \tilde{\sigma}_{ \pm}^{\prime}(\theta-\delta)$ exist (in $\mathbb{R} \cup\{+\infty\}$ ), and furthermore, they must be equal. (Trivially $\tilde{\sigma}_{-}^{\prime}(\theta-) \leq \tilde{\sigma}_{+}^{\prime}(\theta-)$ and for the reverse, note that $\tilde{\sigma}_{+}^{\prime}(\theta-2 \delta) \leq \tilde{\sigma}_{-}^{\prime}(\theta-\delta)$ for $\delta>0$. Letting $\delta \rightarrow 0$ gives $\tilde{\sigma}_{+}^{\prime}(\theta-) \leq \tilde{\sigma}_{-}^{\prime}(\theta-)$.) Denote by $\tilde{\sigma}^{\prime}(\theta-)$ this common value, which we may call the slope of $\tilde{\sigma}$ at $\theta-$. Note that this slope may be $+\infty$.

\section{Proposition 2.7}

Let $f \in S$ and suppose $\theta_{p} \rightarrow \theta$ as $p \rightarrow \infty$. Then

$$
p\left(\theta-\theta_{p}\right) \text { is eventually constant } \Longleftrightarrow \quad \tilde{\sigma}^{\prime}(\theta-)<\infty
$$

in which case $p\left(\theta-\theta_{p}\right)=\Delta(=\sigma(\theta-))$ for $p \geq \tilde{\sigma}^{\prime}(\theta-)$.

Proof. We may write

$$
\tilde{\sigma}(y)=\tilde{\sigma}(x)+\int_{x}^{y} g
$$

for $x<y<\theta$, where $g$ is increasing and locally bounded on $(-\infty, \theta)$. We may take $g$ to be $\tilde{\sigma}_{+}^{\prime}$ or $\tilde{\sigma}_{-}^{\prime}$. Observe that $g(x) \rightarrow 0$ as $x \rightarrow-\infty$, for otherwise $\tilde{\sigma}(y)$ is unbounded as $y \rightarrow-\infty$.

Let $k(\lambda)=p \lambda-\tilde{\sigma}(\lambda)$, where $p$ is fixed but arbitrary. Thus for $\lambda<\mu<\theta$,

$$
k(\mu)-k(\lambda)=\int_{\lambda}^{\mu} p-g(t) d t .
$$

Now suppose $g$ remains bounded near $\theta$. Then, for $p \geq \sup g, g(t)-p \geq 0$ for all $t>0$, so that $k$ is increasing on $(-\infty, \theta)$. As such

$$
p \theta_{p}=\sup _{\lambda<\theta} k(\lambda)=k(\theta-)=p \theta-\tilde{\sigma}(\theta-) .
$$

That is, $p\left(\theta-\theta_{p}\right)$ is constant $(=\Delta)$ for $p \geq g(\theta-)=\tilde{\sigma}^{\prime}(\theta-)$.

Conversely, suppose $g$ is unbounded at $\theta$. Then, no matter how large $p$, there exists $\lambda^{\prime}<\theta$ such that $g\left(\lambda^{\prime}\right)>p$, while $g(\lambda)-p<0$ for $\lambda$ sufficiently small. Hence

$$
\lambda_{p} \stackrel{\text { def }}{=} \inf \{\lambda<\theta: g(\lambda) \geq p\} \quad \text { exists, and } \lambda_{p}<\theta .
$$

As such $k(\lambda)-k\left(\lambda_{p}\right)=\int_{\lambda_{p}}^{\lambda} p-g(t) d t \leq 0$ for $\lambda \geq \lambda_{p}$ and also $\leq 0$ for $\lambda \leq \lambda_{p}$; i.e.

$$
p \theta_{p}=\sup _{\lambda<\theta} h(\lambda)=h\left(\lambda_{p}\right)=p \lambda_{p}-\tilde{\sigma}\left(\lambda_{p}\right) .
$$

Thus for $q>p$,

$$
\begin{aligned}
q\left(\theta--\theta_{q}\right) & =q \theta-q \lambda_{q}+\tilde{\sigma}\left(\lambda_{q}\right)=p \theta+(q-p)\left(\theta-\lambda_{q}\right)-\left[p \lambda_{q}-\tilde{\sigma}\left(\lambda_{q}\right)\right] \\
& \geq p\left(\theta-\theta_{p}\right)+(q-p)\left(\theta-\lambda_{q}\right)>p\left(\theta-\theta_{p}\right),
\end{aligned}
$$

showing that $p\left(\theta-\theta_{p}\right)$ is strictly increasing.

An approach to Dirichlet's Divisor Problem? Proposition 2.7 may be an approach to proving $\theta_{p} \equiv \theta$ in particular cases, where we may be able to judge whether $\tilde{\sigma}^{\prime}(\theta-)<\infty$. For example, if for Dirichlet's divisor problem, we have $\tilde{\sigma}^{\prime}(\theta-)<9$, then

$$
\left.\theta_{p}=\theta-\frac{c}{p} \quad \text { for } p \geq 9-\eta \text { (some constants } c, \eta \text { with } c \geq 0 \text { and } \eta>0\right) \text {. }
$$


But $\theta_{p}=\frac{1}{4}$ for $p \leq 9$, so this would force $c=0$ and hence $\theta=\frac{1}{4}$. In other words, it is enough to prove $\tilde{\sigma}^{\prime}(\theta-)<9$ to settle DDP.

\subsection{The behaviour of $\theta_{p}$ for $p$ small}

The behaviour of $\sigma(\lambda)$ for $\lambda$ large and negative is closely related to the behaviour of $\theta_{p}$ for $p$ small. Since $\sigma(\lambda)$ is increasing and non-negative, so $\lim _{\lambda \rightarrow-\infty} \sigma(\lambda)$ exists, which we denote by $\sigma(-\infty)$. Recall that both $\theta_{p}$ and $p\left(\theta-\theta_{p}\right)$ decrease as $p$ decreases to zero. Thus we may define

$$
\theta_{0}=\lim _{p \rightarrow 0+} \theta_{p} \in[-\infty, \theta] \quad \text { and } \quad \Delta_{0}=\lim _{p \rightarrow 0+} p\left(\theta-\theta_{p}\right) .
$$

Note that $\lim _{p \rightarrow 0+} p \theta_{p}=-\Delta_{0}(\leq 0)$.

\section{Proposition 2.8}

(a) We have $\sigma(-\infty)=\Delta_{0}$.

(b) $\sigma(\lambda)=0$ on $(-\infty, d)$ where $d$ is optimal (i.e. $\sigma(\lambda)>0$ for $\lambda>d$ ) if and only if $\theta_{0}=d$.

Proof. (a), Note that $p \theta_{p} \geq p \lambda-\sigma(\lambda)$ for every $\lambda<\theta$. Let $p \rightarrow 0$ to give $\sigma(\lambda) \geq \Delta_{0}$ for all $\lambda<\theta$. Thus $\sigma(-\infty) \geq \Delta_{0}$. On the other hand,

$$
p \theta_{p}=\sup _{\lambda<\theta}(p \lambda-\sigma(\lambda)) \leq p \theta+\sup _{\lambda<\theta}(-\sigma(\lambda))=p \theta-\sigma(-\infty) .
$$

Thus $\sigma(-\infty) \leq p\left(\theta-\theta_{p}\right)$ and so $\sigma(-\infty) \leq \Delta_{0}$.

(b) $(\Rightarrow)$ If $d=\theta$ the result is trivially true as this is equivalent to $\theta_{p} \equiv \theta$. So we may suppose $d<\theta$. We have

$$
p \theta_{p}=\max \left\{\sup _{\lambda<d}(p \lambda-\sigma(\lambda)), \sup _{d \leq \lambda<\theta}(p \lambda-\sigma(\lambda))\right\}
$$

and so, on the assumption that $\sigma(\lambda)=0$ on $(-\infty, d)$,

$$
\theta_{p}=\max \left\{d, \sup _{d \leq \lambda<\theta}\left(\lambda-\frac{\sigma(\lambda)}{p}\right)\right\} .
$$

For each $\delta>0, \sup _{d+\delta \leq \lambda<\theta}\left(\lambda-\frac{\sigma(\lambda)}{p}\right) \leq \theta-\frac{\sigma(d+\delta)}{p} \rightarrow-\infty$ as $p \rightarrow 0$, so $\theta_{0}=d$ follows.

$(\Leftarrow)$ For the converse implication, suppose $\theta_{0}=d$. Then by monotonicity, $\theta_{p} \geq d$ for all $p$. But $\sup _{\lambda \leq d-\delta}(p \lambda-\sigma(\lambda))<p d$ for every $\delta>0$. Hence

$$
\theta_{p}=\sup _{d-\leq \lambda<\theta}\left(\lambda-\frac{\sigma(\lambda)}{p}\right) .
$$

If $\sigma(d-)>0$, then $\theta_{p} \leq \theta-\frac{\sigma(d-)}{p}$ which is $<d$ for all $p$ sufficiently small, contradicting $\theta_{p} \geq d$. Thus $\sigma(d-)=0$. By the $(\Rightarrow)$ part we cannot have $\sigma(\lambda)=0$ for any $\lambda>d$.

3. Some special classes of functions - I: the case where $f(x)=f([x])$ Let $S_{\text {disc }}$ denote the subset of $S$ of functions $f$ for which $f(x)=f([x])$; i.e. $f$ is of the form

$$
f(x)=\sum_{n \leq x} a_{n}
$$

for some $a_{n}$; indeed $a_{n}=f(n)-f(n-1)$. We can generally say more about $f$ with this extra condition. For example, from Remarks 2.1(iii) we see that $\theta_{p} \geq \theta-\frac{1}{p}$, while in general $p\left(\theta-\theta_{p}\right)$ need not be bounded. In many examples of interest (eg. the general Dirichlet divisor problem) one knows more about the $a_{n}$. 
Here we investigate what can be deduced under the further condition that $a_{n} \ll n^{\varepsilon}$ for every $\varepsilon>0$. The condition of course implies that $\theta \leq 1$. Indeed $\bar{\theta} \leq 1$ since $\sum_{n \leq x}\left|a_{n}\right| \ll x^{1+\varepsilon}$.

Note that $\sigma(\theta-)=\Delta \leq 1$ in this case.

Proposition 3.1

Let $f \in S_{\text {disc }}$ for which $f(n)-f(n-1) \ll n^{\varepsilon}$ for every $\varepsilon>0$ and $\theta>0$. Then $\sigma(\theta-)=\Delta \leq 1-\theta$.

Proof. Let $\lambda<\theta$. There exists $n_{k} \in \mathbb{N}$ such that $n_{k} \nearrow \infty$ and

$$
\left|f\left(n_{k}\right)\right| \geq 2\left(2 n_{k}\right)^{\lambda}
$$

since $\frac{|f(x)|}{x^{\theta-\varepsilon}}$ is unbounded for every $\varepsilon>0$. Let $\delta>0$. Then, for $k$ sufficiently large,

$$
\left|a_{r}\right| \leq r^{\delta} \quad \text { for } r \geq n_{k} .
$$

As such, with $n_{k} \leq t \leq 2 n_{k}$,

$$
\begin{aligned}
|f(t)| & \geq\left|f\left(n_{k}\right)\right|-\left|f(t)-f\left(n_{k}\right)\right| \geq 2\left(2 n_{k}\right)^{\lambda}-\sum_{n_{k}<r \leq t}\left|a_{r}\right| \\
& \geq 2\left(2 n_{k}\right)^{\lambda}-\left(2 n_{k}\right)^{\delta}\left(t-n_{k}\right)
\end{aligned}
$$

for $k$ sufficiently large. Thus for $t \leq n_{k}+\left(2 n_{k}\right)^{\lambda-\delta}$ we have

$$
|f(t)| \geq 2\left(2 n_{k}\right)^{\lambda}-\left(2 n_{k}\right)^{\lambda}=\left(2 n_{k}\right)^{\lambda} \geq t^{\lambda}
$$

if $\theta \geq 0$. Hence $t \in U_{\lambda}\left(n_{k}\right)$ for $n_{k} \leq t \leq n_{k}+\left(2 n_{k}\right)^{\lambda-\delta}$; i.e. $\left|U_{\lambda}\left(n_{k}\right)\right| \geq\left(2 n_{k}\right)^{\lambda-\delta}$. It follows that $1-\sigma(\lambda) \geq \lambda-\delta$ for $\lambda \in(0, \theta)$. This is true for every $\delta>0$, so $1-\sigma(\lambda) \geq \lambda$ for $\lambda \in(0, \theta)$. Hence $\sigma(\theta-) \leq 1-\theta$.

Proposition 3.1 also holds for $f \in S$ if $f(x+y)-f(x) \ll x^{\varepsilon}$ uniformly for $0 \leq y \leq 1$ and any $\varepsilon>0$. For $f \in S_{\text {disc }}$, the condition simplifies to $f(n)-f(n-1) \ll n^{\varepsilon}$.

It is interesting to see to what extent Theorem 2.5 can be proved for the special class of functions in Proposition 3.1. We prove that the only extra condition required is $\sigma(\theta-) \leq 1-\theta$. Furthermore, we show that we may even take $f(n)-f(n-1) \ll \log n$. Note that to prove the equivalent of Theorem 2.5, we only need Theorem 2.4 for $k(\cdot)$ concave, increasing, and bounded by 1 . This in turn makes $k$ strictly increasing (once it is positive) and continuous. We just consider case (b) of Proposition 2.8 as it is of greater interest. The construction resembles that of Theorem 2.4 but now we choose $\tau(x)$ more carefully in order to avoid the large jump at $2^{n}$.

\section{Theorem 3.2}

Let $\phi \in(0,1)$ and $k:[d, \phi] \rightarrow \mathbb{R}$ be strictly increasing and continuous such that $k(d)=0$ and $k(\phi) \leq 1-\phi$. Then there exists $f \in S_{\text {disc }}$ such that $\theta=\phi, f(n)-f(n-1) \ll \log n$, and

$$
\sigma(\lambda)=\left\{\begin{array}{cl}
k(\lambda) & \text { if } d<\lambda \leq \phi \\
0 & \text { if } \lambda \leq d
\end{array}\right.
$$

Proof. First we find $f_{1} \in S$ satisfying the conditions. Let $f_{1}(x)=x^{\tau(x)}$ where $\tau$ is defined on each interval $\left[2^{n}, 2^{n+1}\right]$ by

$$
\tau\left(\frac{3}{2} 2^{n} \pm \frac{\phi-\lambda}{2(\phi-d)} 2^{n(1-k(\lambda))}\right)=\lambda \quad\left(d \leq \lambda \leq \phi, n \in \mathbb{N}_{0}\right)
$$

Observe that $\tau$ is strictly increasing on $\left[2^{n}, \frac{3}{2} 2^{n}\right]$ and strictly decreasing on $\left[\frac{3}{2} 2^{n}, 2^{n+1}\right]$, with minimum and maximum given by $\tau\left(2^{n}\right)=d$ and $\tau\left(\frac{3}{2} 2^{n}\right)=\phi$. Note that $\tau$ is continuous on $(1, \infty)$. 
Clearly, $f_{1} \in S$ with $\theta=\phi$ (so we now replace $\phi$ by $\theta$ ). Also, for $\lambda \in[d, \theta], t \in U_{\lambda}\left(2^{n}\right) \Leftrightarrow \tau(t) \geq \lambda \Leftrightarrow$ $t \in\left[\frac{3}{2} 2^{n}-\frac{\theta-\lambda}{2(\theta-d)} 2^{n(1-k(\lambda))}, \frac{3}{2} 2^{n}+\frac{\theta-\lambda}{2(\theta-d)} 2^{n(1-k(\lambda))}\right]$. Hence

$$
\left|U_{\lambda}\left(2^{n}\right)\right|=\frac{\theta-\lambda}{\theta-d} 2^{n(1-k(\lambda))}
$$

and so $\sigma(\lambda)=1-k(\lambda)$ in this range. As $k(d)=0$ it follows that $\sigma(\lambda)=0$ for $\lambda<d$. It remains to find bounds on $f_{1}(n)-f_{1}(n-1)$. To this end, it is enough to consider $f_{1}(m)-f_{1}(m-1)$ for integers $m$ with $2^{n}<m \leq 2^{n+1}, n \in \mathbb{N}_{0}$. Consider first $m>\frac{3}{2} 2^{n}$, the case when $m \leq \frac{3}{2} 2^{n}$ can be treated similarly. We can write

$$
\begin{aligned}
m & =\frac{3}{2} 2^{n}+\frac{\theta-\lambda_{1}}{2(\theta-d)} 2^{n\left(1-k\left(\lambda_{1}\right)\right)} \\
m-1 & =\frac{3}{2} 2^{n}+\frac{\theta-\lambda_{2}}{2(\theta-d)} 2^{n\left(1-k\left(\lambda_{2}\right)\right)}
\end{aligned}
$$

for some $\lambda_{1}, \lambda_{2}$ such that $d \leq \lambda_{1}<\lambda_{2} \leq \theta$. As such $\tau(m-1)-\tau(m)=\lambda_{2}-\lambda_{1}$. Subtracting the above two equations gives

$$
\left(\theta-\lambda_{1}\right) 2^{n\left(1-k\left(\lambda_{1}\right)\right)}-\left(\theta-\lambda_{2}\right) 2^{n\left(1-k\left(\lambda_{2}\right)\right)}=2(\theta-d) .
$$

Then, since $k\left(\lambda_{2}\right)>k\left(\lambda_{1}\right)$,

$$
\begin{aligned}
\tau(m-1)-\tau(m) & =\left(\theta-\lambda_{1}\right)-\left(\theta-\lambda_{2}\right)=\frac{\left(\theta-\lambda_{1}\right) 2^{n\left(1-k\left(\lambda_{1}\right)\right)}-\left(\theta-\lambda_{2}\right) 2^{n\left(1-k\left(\lambda_{1}\right)\right)}}{2^{n\left(1-k\left(\lambda_{1}\right)\right)}} \\
& \leq \frac{\left(\theta-\lambda_{1}\right) 2^{n\left(1-k\left(\lambda_{1}\right)\right)}-\left(\theta-\lambda_{2}\right) 2^{n\left(1-k\left(\lambda_{2}\right)\right)}}{2^{n\left(1-k\left(\lambda_{1}\right)\right)}}=\frac{2(\theta-d)}{2^{n\left(1-k\left(\lambda_{1}\right)\right)}}
\end{aligned}
$$

by (3.1). But $2^{n} \geq \frac{m}{2}$ and $1-k\left(\lambda_{1}\right) \geq \theta$, so for $m \geq 2$,

$$
|\tau(m)-\tau(m-1)| \leq \frac{2(\theta-d)}{\left(\frac{m}{2}\right)^{\theta}} \leq \frac{4 \theta}{m^{\theta}} .
$$

A similar argument gives the above inequality for $m \leq \frac{3}{2} 2^{n}$ and so (3.2) holds for $m \geq 2$. Thus, for $m \geq 2$,

$$
\begin{aligned}
f_{1}(m)-f_{1}(m-1) & =m^{\tau(m)}-(m-1)^{\tau(m-1)}=m^{\tau(m)}\left\{1-\left(1-\frac{1}{m}\right)(m-1)^{\tau(m-1)-\tau(m)}\right\} \\
& =m^{\tau(m)}\left(1-\left(1+O\left(\frac{1}{m}\right)\right)\left(1+O\left(\frac{\log m}{m^{\theta}}\right)\right)\right) \\
& =O\left(\frac{m^{\tau(m)} \log m}{m^{\theta}}\right)=O(\log m) .
\end{aligned}
$$

Thus $f_{1}$ satisfies the conditions.

Now let $f(x)=f_{1}([x])$. Then $f \in S_{\text {disc }}$ with the same $\theta$ and $f(n)-f(n-1) \ll \log n$ follow immediately. It remains to prove that $f$ has the same $\sigma$-function as $f_{1}$.

First note that for $m \in \mathbb{N}$, we have $m \in U_{\lambda, f}\left(2^{n}\right)$ if and only if $m \in U_{\lambda, f_{1}}\left(2^{n}\right)$, which in turn holds if and only if

$$
m \in\left[\frac{3}{2} 2^{n}-\frac{\theta-\lambda}{2(\theta-d)} 2^{n(1-k(\lambda))}, \frac{3}{2} 2^{n}+\frac{\theta-\lambda}{2(\theta-d)} 2^{n(1-k(\lambda))}\right] \quad(\text { for } d \leq \lambda \leq \theta) .
$$

Let $\varepsilon>0$ and $d<\lambda<\theta$. Then for all sufficiently large $m, m \in U_{\lambda, f}\left(2^{n}\right)$ implies $[m, m+1) \subset U_{\lambda-\varepsilon, f}\left(2^{n}\right)$. For this involves $f(t) \geq t^{\lambda-\varepsilon}$ for every $t \in[m, m+1)$; i.e. $m^{\tau(m)} \geq t^{\lambda-\varepsilon}$. Since $\tau(m) \geq \lambda$, this certainly holds if $m^{\lambda} \geq t^{\lambda-\varepsilon}$. But $t=m+O(1)$ and the RHS is $m^{\lambda-\varepsilon}\left(1+O\left(\frac{1}{m}\right)\right)$, and so it clearly holds for $m$ sufficiently large. Hence

$$
\left|U_{\lambda-\varepsilon, f}\left(2^{n}\right)\right| \geq \sum_{\left|m-\frac{3}{2} 2^{n}\right| \leq \frac{\theta-\lambda}{2(\theta-d)} 2^{n(1-k(\lambda))}} 1 \geq c 2^{n(1-k(\lambda))}
$$

for some $c>0$, and so $\sigma_{f}(\lambda-\varepsilon) \leq k(\lambda)$. This holds for all $\varepsilon>0$, so actually $\sigma_{f}(\lambda-) \leq k(\lambda)$ for $d<\lambda<\theta$. 
For the reverse inequality, we shall need (3.2) for non-integral $m$. In fact using the monotonicity of $\tau$ on both halves of the interval $\left[2^{n}, 2^{n+1}\right]$, one has

$$
\tau(x+y)-\tau(x) \ll \frac{1}{x^{\theta}} \quad \text { uniformly for } y \in[0,1] .
$$

For if $2^{n} \leq x<\frac{3}{2} 2^{n}-1$, then $\tau(x+y)-\tau(x) \leq \tau([x+y]+1)-\tau([x+y])+\tau([x+y])-\tau([x]) \ll x^{-\theta}$. Similarly for $x \in\left[\frac{3}{2} 2^{n}, 2^{n+1}-1\right)$. In the remaining ranges one has (by monotonicity)

$$
|\tau(x+y)-\tau(x)| \leq\left\{\begin{array}{cl}
2\left(\tau\left(\frac{3}{2} 2^{n}\right)-\tau\left(\frac{3}{2} 2^{n}-1\right)\right) & \text { if } \frac{3}{2} 2^{n}-1 \leq x<\frac{3}{2} 2^{n} \\
\max \left\{\tau\left(2^{n+1}+1\right)-\tau\left(2^{n+1}\right), \tau\left(2^{n+1}-1\right)-\tau\left(2^{n+1}\right)\right\} & \text { if } 2^{n+1}-1 \leq x<2^{n+1}
\end{array} .\right.
$$

In either case one has $\tau(x+y)-\tau(x) \ll x^{-\theta}$ uniformly.

Let $\varepsilon>0$ and $d<\lambda<\theta$ as before. Then $U_{\lambda, f}\left(2^{n}\right) \subset U_{\lambda-\varepsilon, f_{1}}\left(2^{n}\right)$ for $n$ sufficiently large. For $t \in S_{\lambda, f}\left(2^{n}\right) \Leftrightarrow m^{\tau(m)} \geq t^{\lambda}$ (where $m=[t]$ ) and $\tau(t)=\tau(m)+O\left(m^{-\theta}\right)$. Thus

$$
t^{\tau(t)} \sim t^{\tau(m)}=(m+O(1))^{\tau(m)} \sim m^{\tau(m)} \geq t^{\lambda},
$$

and so $\tau(t) \geq \lambda-\varepsilon$ for all $t$ (i.e. $n$ ) sufficiently large, and $t \in U_{\lambda-\varepsilon, f_{1}}\left(2^{n}\right)$. Hence

$$
\left|U_{\lambda, f}\left(2^{n}\right)\right| \leq\left|U_{\lambda-\varepsilon, f_{1}}\left(2^{n}\right)\right| \ll 2^{n(1-k(\lambda-\varepsilon))}
$$

and $\sigma_{f}(\lambda) \geq k(\lambda-\varepsilon)$. By continuity of $k$, it follows that $\sigma_{f}(\lambda) \geq k(\lambda)$ for $d<\lambda<\theta$ and hence we must have equality.

\section{Theorem 3.3}

Let $\phi, \phi_{p} \in \mathbb{R}(p>0)$ such that $\phi_{p}$ is increasing and tends to $\phi, \phi \leq 1, p \phi_{p}$ is convex, $\phi_{p} \geq \phi-\frac{1-\phi}{p}$ for all $p$, and $\phi_{p} \rightarrow \phi_{0}>-\infty$ as $p \rightarrow 0+$. Then there exists $f \in S_{\text {disc }}$ with $f(n)-f(n-1) \ll \log n$ such that $\theta=\phi$ and $\theta_{p}=\phi_{p}$.

Proof. The proof follows that of Theorem 2.5 and noting that the condition $\lim _{p \rightarrow 0+} \phi_{p}>-\infty \operatorname{implies}$ that only case (b) of Proposition 2.8 occurs. Thus Theorem 3.2 applies in precisely the same way that Theorem 2.4 applies to Theorem 2.5. The extra condition $\phi_{p} \geq \phi-\frac{1-\phi}{p}$ ensures that $\Delta \leq 1-\phi$.

Example By Theorem 3.2, we can find an $f \in S_{\text {disc }}$ with $\theta \in(0,1)$ satisfying $f(n)-f(n-1) \ll n^{\varepsilon}$ with

$$
\sigma(\lambda)=\left\{\begin{array}{cl}
\sqrt{2 P(\theta-\lambda)} & \text { if } \theta-\frac{1}{2 P}<\lambda<\theta \\
0 & \text { if } \lambda \leq \theta-\frac{1}{2 P}
\end{array}\right.
$$

(some $P>0$ ). This leads to, via Theorem 2.2,

$$
\theta_{p}=\left\{\begin{array}{cc}
\theta-\frac{1}{2 P} & \text { for } p<P \\
\theta-\frac{1}{p}+\frac{P}{2 p^{2}} & \text { for } p \geq P
\end{array}\right.
$$

after some calculation. The example shows quite explicitly that $\theta_{p}$ may be constant on an interval $(0, P)$ of arbitrary length before increasing.

\section{II: Analytic functions}

Let $S_{\text {holo }}$ denote the set of functions $f$ which are holomorphic in a half-strip

$$
\{x+i y: x \geq 1, a \leq y \leq b\}
$$

such that for each $y \in[a, b]$, the function $f_{y}$ defined by $f_{y}(x)=f(x+i y)$ lies in $S$. As such, $\theta_{p}^{(y)}$ and $\theta^{(y)}$ (the " $\theta_{p}$ " and " $\theta$ " for $f_{y}$ respectively) are defined for $a \leq y \leq b$. We note that $\theta^{(y)}$ and $\theta_{p}^{(y)}$ are convex functions of $y$ (for every $p$ ). The convexity of $\theta^{(y)}$ follows from a general result about functions of finite 
order in a strip (see [28], p.180), while the convexity of $\theta_{p}^{(y)}$ follows from the results in [7]. Just as for the 'discrete' case we find the same inequality connecting these. This is a consequence of results in [7] (see Theorem 2 therein).

\section{Proposition 3.4}

With $f \in S_{\text {holo }}$ as above,

$$
\theta^{(y)} \leq \theta_{p}^{(y)}+\frac{1}{p}
$$

for every $y \in(a, b)$.

Proof. It suffices the prove the result for $p \in \mathbb{N}$ since $p\left(\theta^{(y)}-\theta_{p}^{(y)}\right)$ increases with $p$ so the result for $p$ integral implies the same for every $p>0$.

Let $a<y<b$ and $h>0$ chosen sufficiently small so that $a<y-h<y+h<b$. By Cauchy's integral formula

$$
f(x+i y)^{p}=\frac{1}{2 \pi} \int_{0}^{2 \pi} f\left(x+i y+r e^{i \theta}\right)^{p} d \theta
$$

for every $r \in[0, h]$. In particular,

$$
|f(x+i y)|^{p} \leq \frac{1}{2 \pi} \int_{0}^{2 \pi}\left|f\left(x+i y+r e^{i \theta}\right)\right|^{p} d \theta .
$$

Now apply $\int_{0}^{h} \ldots r d r$ to both sides. Thus

$$
\frac{h^{2}}{2}|f(x+i y)|^{p} \leq \frac{1}{2 \pi} \int_{0}^{h} r \int_{0}^{2 \pi}\left|f\left(x+i y+r e^{i \theta}\right)\right|^{p} d \theta d r \leq \frac{1}{2 \pi} \int_{x-h}^{x+h} \int_{y-h}^{y+h}|f(u+i v)|^{p} d u d v .
$$

In particular for $x \geq \frac{h}{2}$,

$$
h^{2}|f(x+i y)|^{p} \leq \frac{1}{\pi} \int_{y-h}^{y+h} \int_{x / 2}^{2 x}|f(u+i v)|^{p} d u d v \ll_{\varepsilon} \int_{y-h}^{y+h} x^{1+p \theta_{p}^{(v)}+\varepsilon} d v \ll x^{1+p \theta_{p}^{\left(y+h^{\prime}\right)}+\varepsilon},
$$

where $\left|h^{\prime}\right| \leq h$. Thus $p \theta^{(y)} \leq 1+p \theta_{p}^{\left(y+h^{\prime}\right)}+\varepsilon$. This holds for every $\varepsilon>0$ and $h$ sufficiently small. Since $\theta_{p}^{(y)}$ is continuous (w.r.t. $y$ ), it follows that $p \theta^{(y)} \leq 1+p \theta_{p}^{(y)}$.

Example The inequality given in Proposition 2.12 is sharp. For let

$$
f(z)=\frac{1}{1-z^{i}+z^{-1}},
$$

where $z^{i}$ is the usual principle value: $\left(r e^{i \theta}\right)^{i}=e^{-\theta} r^{i}$ for $r>0$ and $|\theta|<\pi$. Thus $f$ is well-defined and holomorphic in the cut-plane $\mathbb{C} \backslash(-\infty, 0]$ except at the zeros of $1-z^{i}+\frac{1}{z}$. These zeros are bounded away from 0 since at the zeros we require $\frac{1}{|z|}=\left|1-z^{i}\right| \leq 1+e^{\pi}$. For large $|z|$ the zeros lie roughly on the line $\Im z=-1$. To see this, take absolute values of $z^{i}=1+\frac{1}{z}$ with $z=r e^{i \theta}$ to get

$$
e^{-2 \theta}=1+\frac{2 \cos \theta}{r}+\frac{1}{r^{2}} \text {. }
$$

Thus $\theta=-\frac{\cos \theta}{r}+O\left(\frac{1}{r^{2}}\right)$, and so $z=r-i+O\left(\frac{1}{r}\right)$. It follows that for every $y \neq-1, f(x+i y)$ is well-defined for $x$ sufficiently large. Fix $y>-1$. A straightforward estimation shows that, for large $x$,

$$
\frac{1}{f(x+i y)}=1-x^{i} e^{-\frac{y}{x}}+\frac{1}{x}-\frac{i y}{x^{2}}+O\left(\frac{1}{x^{3}}\right) .
$$

Now

$$
\left|1-x^{i} e^{-\frac{y}{x}}+\frac{1}{x}-\frac{i y}{x^{2}}\right|^{2}=\left(1-e^{-\frac{y}{x}} \cos \log x+\frac{1}{x}\right)^{2}+\left(e^{-\frac{y}{x}} \sin \log x+\frac{y}{x^{2}}\right)^{2}
$$


and $1-e^{-\frac{y}{x}} \cos \log x+\frac{1}{x} \geq \frac{1+y \cos \log x}{x}+O\left(x^{-2}\right) \geq \frac{a}{x}$ for some $a>0$ (depending on $y$ ). Thus $|f(x+i y)| \ll$ $x$. On the other hand, with $x=e^{2 \pi k}(k \in \mathbb{N})$, RHS of $(3.3)=\left(1-e^{-\frac{y}{x}}+\frac{1}{x}\right)^{2}+\frac{y^{2}}{x^{4}}=\frac{(1+y)^{2}}{x^{2}}+O\left(x^{-3}\right)$. Hence $|f(x+i y)| \sim \frac{x}{1+y}$ for such $x$. Thus $\theta^{(y)}=1$ for every $y>-1$. (Similarly we can discuss $y<-1$.)

In order to calculate $\theta_{p}^{(y)}$, we first calculate $\sigma(\lambda)$ and use Lemma 2.3. Fix $y>-1$ and $\lambda<1$. Then $\left|f_{y}(t)\right| \geq t^{\lambda}$ is satisfied if

$$
\left|1-(t+i y)^{i}+\frac{1}{t+i y}\right|^{2} \leq t^{-2 \lambda} .
$$

The LHS above is bounded so, for $\lambda<0,(3.4)$ is always satisfied for large enough $t$. Thus $\left|U_{\lambda}(x)\right|=x$ (for $x$ large) and $\sigma(\lambda)=0$ for $\lambda<0$.

Now consider $0<\lambda<1$. Now $(t+i y)^{i}=t^{i}\left(1-\frac{y}{t}+O\left(\frac{1}{t^{2}}\right)\right)=t^{i}+o(1)$, so (3.4) can only hold if $t=e^{2 \pi n+o(1)}(n \in \mathbb{N})$. Write $t=e^{2 \pi(n+h)}$. Then $t^{i}=e^{2 \pi i h}$ and (after some calculations)

$\left|1-(t+i y)^{i}+\frac{1}{t+i y}\right|^{2}=(1-\cos 2 \pi h)^{2}+(\sin 2 \pi h)^{2}\left(1-\frac{y}{t}\right)+\frac{2}{t}(1-\cos 2 \pi h)(1+y \cos 2 \pi h)+O\left(\frac{1}{t^{2}}\right)$.

This is $\sim 4 \pi^{2} h^{2}$ (whenever $h \succ \frac{1}{t}$ ). Thus (3.4) is satisfied for $h \leq c_{1} t^{-\lambda}$ and fails for $h \geq c_{2} t^{-\lambda}$ (suitable $\left.c_{1}, c_{2}>0\right)$. Hence (3.4) holds in the range $\left[e^{2 \pi\left(n+c_{1} e^{-2 \pi n}\right)}, e^{2 \pi\left(n+c_{2} e^{-2 \pi n \lambda}\right)}\right] \approx\left[e^{2 \pi n}+O(1), e^{2 \pi n}+\right.$ $\left.c^{\prime} e^{2 \pi(1-\lambda) n}\right]$ for some $c^{\prime}>0$. Hence $\left|U_{\lambda}\left(e^{2 \pi n}\right)\right| \asymp e^{2 \pi(1-\lambda) n}$, so that $\sigma(\lambda)=\lambda$. That is,

$$
\sigma(\lambda)= \begin{cases}\lambda & \text { if } 0<\lambda<1 \\ 0 & \text { if } \lambda \leq 0\end{cases}
$$

Now Theorem 2.2 gives

$$
\theta_{p}=\left\{\begin{array}{cl}
0 & \text { if } 0<p<1 \\
1-\frac{1}{p} & \text { if } p \geq 1
\end{array} .\right.
$$

Thus $\theta^{(y)}=\theta_{p}^{(y)}+\frac{1}{p}$ for the range $p \geq 1$ and every $y>-1$.

The problem whether Theorems 2.4 and 2.5 extend to the holomorphic case remains open.

\section{The abscissae of the associated Mellin tranform}

\subsection{Lindelöf functions}

Formally the Mellin transform of a function $f:(0, \infty) \rightarrow \mathbb{C}$ is defined by

$$
\hat{f}(s)=\int_{0}^{\infty} x^{-s} d f(x)
$$

We shall assume that $f \in S$ and that $f(x)=0$ for $x<1$, as this covers most cases of interest. As such, the Mellin transform is well-defined for $\sigma=\Re s>\theta$. For

$$
\int_{0}^{X} x^{-s} d f(x)=\frac{f(X)}{X^{s}}+s \int_{1}^{X} \frac{f(x)}{x^{s+1}} d x .
$$

The first term on the right tends to 0 as $X \rightarrow \infty$ whenever $\sigma>\theta$ while the integral converges absolutely for $\sigma>\theta_{1}$. In particular, for $\sigma>\theta$

$$
\hat{f}(s)=s \int_{1}^{\infty} \frac{f(x)}{x^{s+1}} d x
$$

and by standard results of complex analysis, $\hat{f}$ is holomorphic in this region. However the RHS of (4.1) converges absolutely for $\sigma>\theta_{1}$ and so $\hat{f}$ has an automatic analytic continuation to the half-plane $H_{\theta_{1}}{ }^{4}$ and (4.1) holds for $\sigma>\theta_{1}$. We shall denote any analytic continuation of $\hat{f}$ beyond $H_{\theta_{1}}$ by $\hat{f}$.

\footnotetext{
${ }^{4} H_{\alpha}=\{z \in \mathbb{C}: \Re z>\alpha\}$.
} 
We see that, for $\sigma>\theta_{1}$ at least, $\hat{f}$ is of finite order: i.e. $\hat{f}(\sigma+i t)=O\left(t^{A}\right)$ for some $A$ (indeed we may take $A=1$ here). As such we can define the usual 'Lindelöf' functions (wherever they make sense):

$$
\begin{aligned}
\mu(\sigma) & =\inf \left\{\lambda: \hat{f}(\sigma+i t)=O\left(t^{\lambda}\right) \text { for } t \geq 1\right\} \\
\mu_{p}(\sigma) & =\inf \left\{\lambda:\left(\frac{1}{T} \int_{T}^{2 T}|\hat{f}(\sigma+i t)|^{p} d t\right)^{1 / p}=O\left(T^{\lambda}\right)\right\} .
\end{aligned}
$$

Occasionally, we may write $\mu_{\hat{f}}(\sigma)$ and $\mu_{\hat{f}, p}(\sigma)$ for these functions to show the dependence on $\hat{f}$. These are examples of the 'analytic case' from section 3II.

From above we automatically have $\mu(\sigma) \leq 1$ for $\sigma>\theta_{1}$.

\section{Proposition 4.1}

Let $f \in S$. Then $\mu(\cdot)$ and $\mu_{p}(\cdot)$ are convex for every $p$. Furthermore, if $\bar{\theta}<\infty$ and $f(1) \neq 0$ then both functions are zero for $\sigma>\bar{\theta}$. Consequently both are decreasing functions of $\sigma$.

Proof. The convexity of $\mu(\sigma)$ follows from a general result about functions of finite order in a vertical strip (see [28], p.180), while the convexity of $\mu_{p}(\sigma)$ follows from the results in [7].

For the second part, we have for $\sigma>\bar{\theta}$

$$
|\hat{f}(s)| \leq \int_{1-}^{\infty} x^{-\sigma} d V_{f}(x)=\hat{V}_{f}(\sigma)
$$

so that $\mu(\sigma) \leq 0$ for $\sigma>\bar{\theta}$. But for $\sigma$ sufficiently large we have

$$
|\hat{f}(\sigma+i t)|=\left|f(1)+\int_{1}^{\infty} x^{-s} d f(x)\right| \geq|f(1)|-\int_{1}^{\infty} x^{-\sigma} d V_{f}(x) .
$$

The final integral tends to zero as $\sigma \rightarrow \infty$, hence if $f(1) \neq 0$, then $|\hat{f}(\sigma+i t)| \geq \frac{1}{2}|f(1)|$ for $\sigma$ sufficiently large and all $t \in \mathbb{R}$. Thus $\mu(\sigma), \mu_{p}(\sigma) \geq 0$ for $\sigma$ sufficiently large (all $p$ ). By convexity, we must have $\mu(\sigma)=\mu_{p}(\sigma)=0$ for $\sigma>\bar{\theta}$, and both functions are decreasing.

Slightly more generally, if $f$ is zero on $(0, c)$ but $f(c) \neq 0$ for some $c>1$, then the same conclusions hold. In other cases ${ }^{5}$ it may be that $\mu(\sigma)$ is negative for all $\sigma$. It follows immediately from the convexity that (under the conditions of Proposition 4.1)

$$
\mu(\sigma) \leq \frac{\bar{\theta}-\sigma}{\bar{\theta}-\theta_{1}} \quad \text { for } \theta_{1}<\sigma \leq \bar{\theta} .
$$

Proposition 3.4 can be applied to $\hat{f}$ to give:

$$
\mu(\sigma) \leq \mu_{p}(\sigma)+\frac{1}{p}
$$

for every $p>0$ and $\sigma>\theta_{1}$. Furthermore, the result remains true whenever $\hat{f}$ has an analytic continuation to $H_{\alpha}$ for some $\alpha<\theta_{1}$ of finite order.

This inequality is sharp (at least for the case $p=2$ ) as an example of Kahane shows (see [19], Theorem 2.2). For his example, $\mu(\sigma)=\mu_{2}(\sigma)+\frac{1}{2}$ for a range of values of $\sigma$. See $\S 5$ (example (vii)) for more details.

4.2 Connection between $\theta_{2}$ and $\mu_{2}(\sigma)$

As for the abscissae in section 1, determining the Lindelöf functions is often exceedingly difficult. We aim to see to what extent the abscissae of a function are connected to the abscissae of its Mellin transform.

${ }^{5}$ For example, if $f(x)=x-1$ for $x \geq 1$, then $\hat{f}(s)=\frac{1}{s-1}$ and $\mu(\sigma) \equiv-1$. 
First we prove a connection between $\theta_{2}$ and $\mu_{2}$. At the heart of this connection lies Parseval's equality.

\section{Theorem 4.2}

Let $f \in S$ with $\theta_{2}, \hat{f}$ and $\mu_{2}(\sigma)$ defined as before and suppose $\theta_{2}>-\infty$. Then $\mu_{2}\left(\theta_{2}+\right) \leq \frac{1}{2}$.

Furthermore, suppose that $\hat{f}$ has an analytic continuation of finite order to $H_{\alpha}$ for some $\alpha<\theta_{2}$. Then $\mu_{2}\left(\theta_{2}\right)=\frac{1}{2}$.

Note: in particular, if $\theta_{1}<\theta_{2}$ then the extra analyticity assumption is satisfied automatically with $\alpha=\theta_{1}$.

Proof. Put $g(s)=\int_{1}^{\infty} \frac{f(x)}{x^{s+1}} d x$. Thus $g(s)=\hat{f}(s) / s$ for $\sigma>\theta_{1}$. Let $\nu$ denote the infimum of $\sigma$ for which $\int_{-\infty}^{\infty}\left|g_{\sigma}\right|^{2}$ converges. By an argument identical to that given in Theorem 12.5 of [29], we have $\nu=\theta_{2}$ and

$$
\frac{1}{2 \pi} \int_{-\infty}^{\infty}|g(\sigma+i t)|^{2} d t=\int_{0}^{\infty} \frac{|f(x)|^{2}}{x^{2 \sigma+1}} d x
$$

for $\sigma>\theta_{2}$ (see also Theorem 71 of [30]). As such, for $\sigma>\theta_{2}$,

$$
\int_{T}^{2 T}|\hat{f}(\sigma+i t)|^{2} d t \asymp T^{2} \int_{T}^{2 T}\left|\frac{\hat{f}(\sigma+i t)}{\sigma+i t}\right|^{2} d t=o\left(T^{2}\right)
$$

and so $\mu_{2}(\sigma) \leq \frac{1}{2}$ for every $\sigma>\theta_{2}$; i.e. $\mu_{2}\left(\theta_{2}+\right) \leq \frac{1}{2}$ as required.

Now assume that $\hat{f}$ has an analytic continuation of finite order to $H_{\alpha}$ for some $\alpha<\theta_{2}$. that

Suppose, for a contradiction, that $\mu_{2}\left(\theta_{2}\right)<\frac{1}{2}$. Then, by continuity of $\mu_{2}$, there exists $\sigma<\theta_{2}$ such

$$
\int_{T}^{2 T}|\hat{f}(\sigma+i t)|^{2} d t \ll T^{2-\delta}
$$

for some $\delta>0$. But then

$$
\int_{T}^{2 T}|g(\sigma+i t)|^{2} d t \asymp \frac{1}{T^{2}} \int_{T}^{2 T}|\hat{f}(\sigma+i t)|^{2} d t=O\left(T^{-\delta}\right) .
$$

By telescoping it follows that the LHS of (4.3) is finite; i.e. $\sigma \geq \nu$. This contradicts the fact that $\sigma<\nu\left(=\theta_{2}\right)$.

\section{Remarks 4.1}

(i) The analyticity condition of Theorem 4.2 is necessary if we want $\mu_{2}\left(\theta_{2}\right)=\frac{1}{2}$ to hold. For example, taking $f(x)=[x]$, then $\theta_{2}=1$. In this case $\hat{f}(s)=\zeta(s), \mu(\sigma)$ is the usual Lindelöf function for $\zeta(s)$ and $\mu_{2}$ is given by

$$
\mu_{2}(\sigma)=\left\{\begin{array}{cc}
0 & \text { if } \sigma \geq \frac{1}{2} \\
\frac{1}{2}-\sigma & \text { if } \sigma \leq \frac{1}{2}
\end{array} .\right.
$$

Theorem 4.2 just says $\mu_{2}(1) \leq \frac{1}{2}$, whereas we know $\mu_{2}(1)=0$.

As we only have one pole it is easy to adjust the example to make the Mellin transform holomorphic to the left of $\theta_{1}$ whilst keeping $\mu$ and $\mu_{2}$ the same. In the above example, one could take $f_{1}(x)=$ $[x]-x$. As such, $\hat{f}_{1}(s)=\zeta(s)-\frac{s}{s-1}$ which is entire. Now $\theta_{2}=0$, while $\mu, \mu_{2}$ remain the same as above. Theorem 4.2 now says $\mu_{2}(0)=\frac{1}{2}$.

The same procedure can be used if we have a finite number of poles. However with an infinite number of poles we run into difficulty. For example, take $f(x)=M(x)=\sum_{n \leq x} \mu(n)$, where $\mu(n)$ is the Möbius function. It is well-known that

$$
\theta=\Theta:=\sup \{\Re \rho: \zeta(\rho)=0\} .
$$

Further, if $\int_{0}^{x}|M|=O\left(x^{1+\alpha}\right)$ for some $\alpha<1$, then $\int_{1}^{\infty} \frac{M(x)}{x^{s+1}} d x$ converges (absolutely) for $\sigma>\alpha$ and $\frac{1}{\zeta(s)}$ is holomorphic and $\zeta(s) \neq 0$ for $\sigma>\alpha$. i.e. we must have $\theta_{1} \geq \Theta$. This implies $\theta_{p} \equiv \Theta$. 
(ii) The Babenko-Beckner inequality (Haussdorff-Young for Fourier transforms - see [2]) gives $\mu_{q}\left(\theta_{p}+\right) \leq$ $\frac{1}{p}$ for $1<p \leq 2$, where $\frac{1}{p}+\frac{1}{q}=1$. This interpolates the inequalities $\mu\left(\theta_{1}+\right) \leq 1$ and $\mu_{2}\left(\theta_{2}+\right) \leq \frac{1}{2}$. However, unlike the $p=2$ case, in general one doesn't have equality.

4.3. Further Inequalities for $\mu(\sigma)$ and $\mu_{2}(\sigma)$ and the Extremal case

The convexity of $\mu$ and $\mu_{2}$ together with Theorem 4.2 and (4.2) give further inequalities for these functions. This is perhaps best seen with the aid of a diagram.

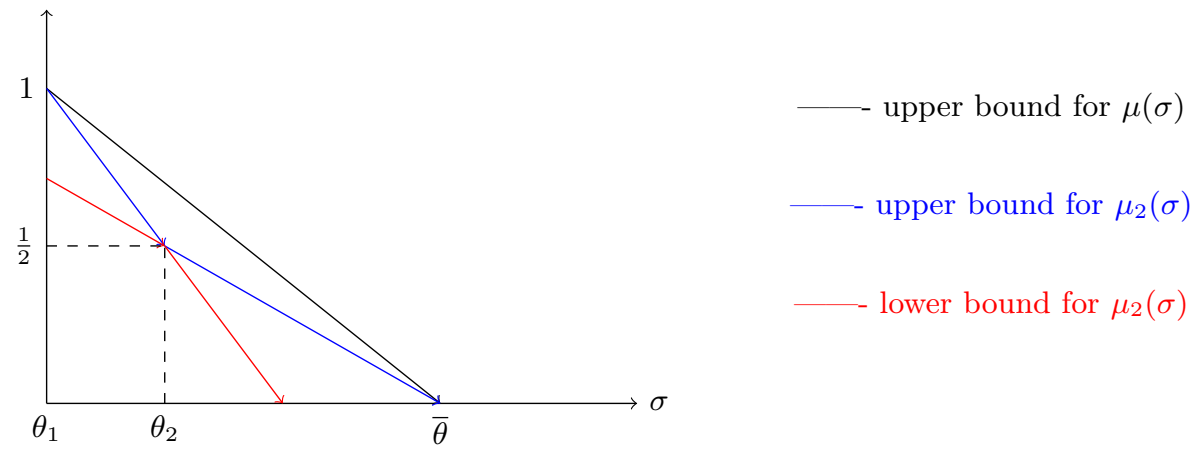

From Theorem 4.2 and (4.2) we must have (given the conditions)

$$
\frac{1}{2}=\mu_{2}\left(\theta_{2}\right) \leq \mu\left(\theta_{2}\right) \leq \frac{\bar{\theta}-\theta_{2}}{\bar{\theta}-\theta_{1}} \quad\left(\text { provided } \theta_{1}<\bar{\theta}<\infty\right) .
$$

In particular, if the left and right hand sides are equal (i.e. $2 \theta_{2}=\theta_{1}+\bar{\theta}$ ) we must have equality throughout. But $2 \theta_{2} \leq \theta_{1}+\theta$, so this is only possible if $\bar{\theta}=\theta$. As such, convexity forces $\mu(\sigma)$ equal to the upper bound on $\left(\theta_{1}, \bar{\theta}\right)$ in (4.2); i.e. the blue, red, and black lines coincide. This leads to:

\section{Corollary 4.3}

Suppose that $\theta_{1}<\theta=\bar{\theta}$ and $2 \theta_{2}=\theta_{1}+\theta$. Then $\mu_{p} \equiv \mu$ for every $p>0$ on $\left(\theta_{1}, \infty\right)$ and

$$
\mu(\sigma)=\left\{\begin{array}{cl}
\frac{\theta-\sigma}{\theta-\theta_{1}} & \text { if } \theta_{1}<\sigma<\theta \\
0 & \text { for } \sigma \geq \theta
\end{array} .\right.
$$

We shall call this case extremal. The case where $\theta_{p} \equiv \theta=\bar{\theta}$ can be thought of as a degenerate extremal case, since now the interval $\left(\theta_{1}, \bar{\theta}\right)$ is empty and Corollary 4.3 says nothing.

Note that in the extremal case, convexity of $p \theta_{p}$ forces $p\left(\theta-\theta_{p}\right)$ to be constant for $p \geq 1$. Furthermore, the condition $2 \theta_{2}=\theta_{1}+\theta$ is equivalent to $\theta-\theta_{1}=2\left(\theta-\theta_{2}\right)$, which in turn is easily seen to be equivalent to $\sigma(\theta-\lambda) \geq \sigma(\theta-)-\lambda$ and forces $\sigma(\theta-)=\theta-\theta_{1}$. This case shows that it is possible for $\mu_{p} \equiv \mu$ even though $\theta_{p} \not \equiv \theta$.

\section{Examples}

(i) Let $f(x)=\{x\}$ (for $x \geq 1$ ), where $\{x\}$ denotes the fractional part of $x$. For this function $\theta_{p} \equiv \theta=0$. Also $V_{f}(x) \sim 2 x$ since the total variation is 2 for each interval $(n, n+1]$. Hence $\bar{\theta}=1$. In this case $\sigma(\lambda) \equiv 0$ trivially as $\left|U_{\lambda}(x)\right| \sim x$ for all $\lambda<0$.

The Mellin transform is

$$
\hat{f}(s)=\frac{s}{s-1}-\zeta(s)
$$

which is entire of finite order. The mean values of $\zeta$ imply that

$$
\mu_{2}(\sigma)=\left\{\begin{array}{cc}
0 & \text { if } \sigma \geq \frac{1}{2} \\
\frac{1}{2}-\sigma & \text { if } \sigma<\frac{1}{2}
\end{array}\right.
$$

and by the functional equation it is also known that $\mu(\sigma)=\mu_{2}(\sigma)$ for $\sigma \geq 1$ and $\sigma \leq 0$. What happens inside the critical strip $(0<\sigma<1)$ is still unknown. The Lindelöf Hypothesis (LH) is that $\mu(\sigma)=\mu_{2}(\sigma)$ here. This is equivalent to $\mu_{p} \equiv \mu$ on $\mathbb{R}$. The fourth power moment of $\zeta$ is known and gives $\mu_{4} \equiv \mu_{2}$. So $\mu_{p} \equiv \mu_{2}$ for $0<p \leq 4$ follows. 
(ii) (Dirichlet's divisor problem). Let $\Delta(x)$ be defined by

$$
\Delta(x)=\sum_{n \leq x}(d(n)-\log n-2 \gamma)
$$

where $d(n)$ is the number of divisors of $n$ and $\gamma$ is Euler's constant. The best bounds on $\theta$ are $\frac{1}{4} \leq \theta \leq \frac{131}{416}$. It is conjectured that $\theta=\frac{1}{4}$.

Regarding $\theta_{p}$, it is known that $\theta_{p}=\frac{1}{4}$ for $0<p \leq 9$ (at least), see [15], [11]. As for $\sigma(\lambda)$, by Proposition 2.8(b), $\sigma(\lambda)=0$ for $\lambda<\frac{1}{4}$, while $\sigma(\theta-) \leq 1-\theta$.

More generally, take $f(x)=\Delta_{k}(x)$ where

$$
\Delta_{k}(x)=\sum_{n \leq x}\left(d_{k}(n)-P_{k}(\log n)\right) .
$$

Here $d_{k}(n)$ is the coefficient of $n^{-s}$ in the Dirichlet series for $\zeta(s)^{k}$ and $P_{k}$ is a polynomial of degree $k-1$, suitably chosen so that the associated Mellin transform is entire. Then $\theta=\alpha_{k}$ and $\theta_{2}=\beta_{k}$ (using the notation of Titchmarsh [29]). The conjecture is that (for every $k$ )

$$
\alpha_{k} \stackrel{(i)}{=} \beta_{k} \stackrel{(i i)}{=} \frac{1}{2}-\frac{1}{2 k} .
$$

Note that (ii) (for all $k$ ) is equivalent to the Lindelöf Hypothesis, while (i) and (ii) together are not known to follow from LH or even RH. Equality (i) (without (ii)) would just be saying that $\theta_{p} \equiv \theta$. As for the corresponding Lindelöf functions, we have $\mu(\sigma)=k \mu_{\zeta}(\sigma)$ while $\mu_{p}(\sigma)=\mu_{\zeta^{k}, p}(\sigma)$. These functions are not known for any value inside the critical strip except when $k=1,2$ and $p \leq 2$. On $\mathrm{LH}$, all these functions are equal; namely

$$
\mu_{p}(\sigma) \equiv \mu(\sigma)=\left\{\begin{array}{cc}
0 & \text { if } \sigma \geq \frac{1}{2} \\
k\left(\frac{1}{2}-\sigma\right) & \text { if } \sigma<\frac{1}{2}
\end{array} .\right.
$$

(iii) Let

$$
f(x)=\left\{\begin{array}{cl}
\left|\zeta\left(\frac{1}{2}+i x\right)\right|^{2} & \text { for } x \geq 1 \\
0 & \text { otherwise }
\end{array} .\right.
$$

For this function, $\theta=2 \mu_{\zeta}\left(\frac{1}{2}\right)$ where $\mu_{\zeta}$ is the Lindelöf function for $\zeta(s)$. It is known that $\mu_{\zeta}\left(\frac{1}{2}\right) \leq \frac{32}{205}$ (see [14]) and, on LH, $\theta=0$. Here, $\theta_{1}=\theta_{2}=0$ on account of the second and fourth power moments of $\zeta\left(\frac{1}{2}+i x\right)$.

Notice that $\zeta\left(\frac{1}{2}+i x\right)^{2} \in S_{\text {holo }}$ since $\zeta(y+i x)^{2} \in S$ for each fixed $y$. By Proposition 3.4, $\theta \leq \theta_{p}+\frac{1}{p}$ and so $\theta_{p} \rightarrow \theta$. The Lindelöf Hypothesis is the statement that $\theta_{p}$ is constant.

Also for this function, $\bar{\theta} \leq 1$. For $f^{\prime}(x)=-2 \Im\left(\zeta^{\prime}\left(\frac{1}{2}+i x\right) \zeta\left(\frac{1}{2}-i x\right)\right)$ and so, by Cauchy-Schwarz,

$$
V_{f}(x) \leq 2 \sqrt{\int_{1}^{x}\left|\zeta^{\prime}\left(\frac{1}{2}+i t\right)\right|^{2} d t \int_{1}^{x}\left|\zeta\left(\frac{1}{2}+i t\right)\right|^{2}} d t \sim \frac{2}{\sqrt{3}} x(\log x)^{2} .
$$

Presumably $\bar{\theta}=1$. The Mellin transform of this function has been studied by a number of authors (starting with [16]) and holomorphic properties and various bounds on the associated Lindelöf functions are known. Thus, using the notation of [16],

$$
\hat{f}(s)=s \int_{1}^{\infty} \frac{\left|\zeta\left(\frac{1}{2}+i x\right)\right|^{2}}{x^{s+1}} d x=s Z_{1}(s+1) .
$$

It is known (see [17]) that $Z_{1}$ has an analytic continuation to the whole plane except for a double pole at $s=1$ and singularities which are at most simple poles at $-1,-3,-5, \ldots$. Thus $\hat{f}$ has an analytic continuation to the whole plane except for a simple pole at $s=0$ with residue 1 , and at most simple poles at $-2,-4,-6, \ldots$. 
Regarding the Lindelöf functions, the relation $\mu(\sigma)=1+\mu_{Z_{1}}(\sigma+1)$ (and similarly for $\mu_{2}(\sigma)$ ) and using known bounds (see [17] and [18]) we have

$$
\mu(\sigma) \leq \begin{cases}\frac{2-4 \sigma}{3} & \text { for }-1 \leq \sigma \leq-\frac{1}{2} \\ \frac{5}{6}-\sigma & \text { for }-\frac{1}{2} \leq \sigma \leq 0\end{cases}
$$

and $\mu_{2}(\sigma) \leq-2 \sigma$ for $-1 \leq \sigma \leq-\frac{1}{2}$ while

$$
\mu_{2}(\sigma)=\frac{1}{2}-\sigma \text { for }-\frac{1}{2} \leq \sigma \leq 0
$$

Note that this last equality contains the value $\mu_{2}(0)=\frac{1}{2}$ from $^{6}$ Theorem 4.2. Ivic [17] suggested that $\mu(\sigma)=\mu_{2}(\sigma)$ for $-\frac{1}{2} \leq \sigma \leq 0$ (at least). Perhaps $\mu_{2} \equiv \mu$ here.

(iv) Another example comes from Bernoulli convolutions. For $\rho>1$, let

$$
C(x)=\#\left\{\varepsilon_{1}+\varepsilon_{2} \rho+\cdots+\varepsilon_{n} \rho^{n-1} \leq x: \varepsilon_{i} \in\{0,1\}, n \in \mathbb{N}\right\} .
$$

One finds that $C(x) \asymp x^{\tau}$ where $\tau=\frac{\log 2}{\log \rho}$. Furthermore $C\left(\rho^{n} x\right) \sim 2^{n} \mu[0, x]$, where $\mu$ is the unique probability measure satisfying $\mu(E)=\frac{1}{2} \mu(\rho E)+\frac{1}{2} \mu(\rho E-1)$ (see [12]). It is known that $\mu$ is either absolutely continuous or purely singular, and the problem is to decide which. It is known to be singular for $\rho$ a Pisot number (as shown by Erdős) and also for $\rho>2$. A major breakthrough came in 1995 when Solomyak [26] proved that $\mu$ is absolutely continuous with an $L^{2}$-density for almost all $\rho \in(1,2]$.

Now let $f(x)=C(x)-C(x-1 / \rho)$. In [12], it was shown that $\int^{x} f \asymp x^{\tau}$, so $\theta_{1}=\tau-1$, while $\int^{x} f^{2} \ll x^{2 \tau-1}$ was shown to be equivalent to $\mu$ being absolutely continuous and having an $L^{2}$ density (this was based on a criterion by Kahane and Salem [20]). Thus by Solomyak's result, $\theta_{2}=\tau-1$ for almost all $\rho \in(1,2]$. Furthermore, $\bar{\theta}=\tau$ (since for every number of the form $\varepsilon_{1}+\varepsilon_{2} \rho+\cdots+\varepsilon_{n} \rho^{n-1}$ there is a jump of one at least) while

$$
\theta \leq \tau\left(1-\frac{1}{n}\right) \quad \text { for } n-1<\tau \leq n \text { where } n \in \mathbb{N} .
$$

(Theroem 1.5 [12]). In particular $\theta_{p} \equiv \theta$ if $\tau \in \mathbb{N}$ (i.e. $\rho$ is an $n^{\text {th }}$-root of 2). But for $\rho=\frac{1+\sqrt{5}}{2}$ (a Pisot number) it was shown that $\theta \geq \frac{\tau}{3}>\tau-0.9603$, so in this case $\theta_{p}<\theta$ for all $p$.

An open problem is then: for which $\rho$ do we have $\theta_{p} \equiv \theta$ ?

(v) Let $f(x)=\sin \left(e^{x}\right)$ for $x \geq 1$ and zero otherwise. In this case $\theta_{p} \equiv \theta=0$ while $\bar{\theta}=\infty$. For $1 \geq|f(x)| \not \nrightarrow 0$ while $\int_{0}^{x} f^{2}=\int_{1}^{x}\left(\sin \left(e^{t}\right)\right)^{2} d t=\int_{e}^{e^{x}} \frac{1-\cos 2 y}{2} d y=\frac{x}{2}+O(1)$. These imply $\theta=0=\theta_{2}$. Noting that $\int_{0}^{\infty}|f|^{p}=\infty$ gives $\theta_{p} \equiv 0$. On the other hand

$$
V_{f}(x)=\int_{0}^{x} e^{t}\left|\cos \left(e^{t}\right)\right| d t=\int_{1}^{e^{x}}|\cos u| d u \asymp e^{x},
$$

which is exponentially large. Thus $\bar{\theta}=\infty$.

The Mellin transform is

$$
\hat{f}(s)=s \int_{1}^{\infty} \frac{\sin \left(e^{x}\right)}{x^{s+1}} d x=s \int_{e}^{\infty} \frac{\sin y}{y(\log y)^{s+1}} d y .
$$

This integral converges absolutely for $\sigma>0$. Integrating by parts gives (for $\sigma>0$ )

$$
\hat{f}(s)=s \frac{\cos e}{e}-s(s+1) \int_{e}^{\infty} \frac{\cos y}{y^{2}(\log y)^{s+2}} d y-s \int_{e}^{\infty} \frac{\cos y}{y^{2}(\log y)^{s+1}} d y,
$$

showing that $\hat{f}$ is entire, with $\hat{f}(\sigma+i t)=O\left(t^{2}\right)$. Thus $\mu_{2}(\sigma)$ and $\mu(\sigma)$ are constant (being convex and bounded). Since $\mu_{2}\left(\theta_{2}\right)=\mu_{2}(0)=\frac{1}{2}$, it follows that $\mu_{2}(\sigma) \equiv \frac{1}{2}$.

Further, from $(5.1) \mu(\sigma) \equiv$ constant $\in\left[\frac{1}{2}, 1\right]$. Which constant?

\footnotetext{
${ }^{6}$ Actually Theorem 4.2 would only give $\leq$ here. To get equality we need to cancel off the simple pole at 0 . This can be done by considering instead $f(x)=\left|\zeta\left(\frac{1}{2}+\overline{i x}\right)\right|^{2}-\log x$, which keeps all the abscissa the same.
} 
(vi) Let $f \in S_{\text {disc }}$ be defined by $f(x)=f_{1}([x]), f_{1}(x)=x^{\tau(x)}$ and $\tau(\cdot)$ is given on each interval $\left[2^{n}, 2^{n+1}\right]$ by

$$
\tau\left(\frac{3}{2} 2^{n} \pm \lambda 2^{\left(\lambda+\frac{1}{2}\right) n}\right)=\frac{1}{2}-\lambda \quad\left(0 \leq \lambda \leq \frac{1}{2}, n \in \mathbb{N}_{0}\right) .
$$

After Theorem 3.2, $f$ satisfies $f(n)-f(n-1) \ll \log n, \bar{\theta}=\theta=\frac{1}{2}$ and $\nu(\lambda)=\lambda+\frac{1}{2}$ for $0<\lambda<\frac{1}{2}$ while $\nu(\lambda)=1$ for $\lambda \geq \frac{1}{2}$. This leads easily to

$$
\theta_{p}=\left\{\begin{array}{cc}
\frac{1}{2}-\frac{1}{2 p} & \text { if } p>1 \\
0 & \text { if } p \leq 1
\end{array} .\right.
$$

This is an 'extremal' case as in Corollary 4.3. As such we have

$$
\mu_{p}(\sigma) \equiv \mu(\sigma)=\left\{\begin{array}{cl}
1-2 \sigma & \text { if } 0<\sigma<\frac{1}{2} \\
0 & \text { if } \sigma \geq \frac{1}{2}
\end{array} .\right.
$$

This is an example where $f(x)=\sum_{n \leq x} a_{n}$ where $a_{n} \ll \log n$, and $\theta_{p}, \theta, \mu_{p}(\sigma), \mu(\sigma)$ are explicitly known and $\theta_{p}<\theta$ while $\mu_{p} \equiv \mu$.

(vii) (Kahane's example). In [19] (Theorem 2.2), Kahane proves the existence of a Dirichlet series of the form $\sum_{n=1}^{\infty} \varepsilon_{n}\left((2 n-1)^{-s}-(2 n)^{-s}\right)$ with $\varepsilon_{n}= \pm 1$ for which

$$
\mu(\sigma)=\left\{\begin{array}{cl}
1-\sigma & \text { for } 0<\sigma<1 \\
0 & \text { for } \sigma \geq 1
\end{array} \quad \text { while } \quad \mu_{2}(\sigma)=\left\{\begin{array}{cl}
\frac{1}{2}-\sigma & \text { for } 0<\sigma<\frac{1}{2} \\
0 & \text { for } \sigma \geq \frac{1}{2}
\end{array} .\right.\right.
$$

(Actually, Kahane proved the formula for $\mu(\sigma)$, but the formula for $\mu_{2}(\sigma)$ is easily proven using methods from, say, [23].) Let $f(x)=\sum_{n \leq x} a_{n}$ where $a_{2 k-1}=-a_{2 k}=\varepsilon_{k}$. Then $|f(x)|=1$ on intervals $[2 k-1,2 k)$ and zero on $[2 k, 2 k+1)$. It readily follows that $\theta_{p} \equiv \theta=0$, while $\bar{\theta}=1$. This is an example of an $f \in S_{\text {disc }}$ where $\theta_{p} \equiv \theta$ while $\mu_{p}<\mu$.

Final comments Many of these examples point to $\mu_{2} \equiv \mu$ (and hence $\mu_{p} \equiv \mu$ ), through proofs or conjectures. It suggests that $\mu_{2} \equiv \mu$ in quite general circumstances, though not in all as shown by Kahane's example and the example at the end of $\S 3$. Deciding what mechanism makes $\mu_{2} \equiv \mu$ is likely to be extremely difficult.

\section{The case $\theta=\infty$}

There is an interesting class of functions which are not of polynomial growth yet $\theta_{p}$ is finite for all $p$. Examples are $d\left(\left[e^{x-1}\right]\right)$ (where $d$ is the divisor function) and, conjecturally, $\zeta\left(\frac{1}{2}+i e^{x}\right)$. These will be discussed later. We shall see that much of the previous theory extends to this case. Some results extend without trouble but extra care is needed when earlier we relied on $\theta$ being finite.

Let $S_{\infty}$ denote the space of functions $f:(0, \infty) \rightarrow \mathbb{C}$ for which (i) $f(x)=0$ for $x<1$ and (ii) $f$ has bounded variation on any bounded interval, but for which $\int_{0}^{x}|f|^{p}=O\left(x^{A}\right)$ for every $p>0$ (for some $A$, dependent on $p$ ) but $f(x)=\Omega\left(x^{A}\right)$ for every $A$.

The proof of Proposition 1.1 shows that both (a), (c), and (d) of Proposition 1.1 remain true for $f \in S_{\infty}$; namely, $\theta_{p}$ increases with $p$ and $p \theta_{p}$ is a convex function of $p$.

Further, we may again define $U_{\lambda}(x)$ and $\sigma(\lambda)$ as before, this time for all $\lambda \in \mathbb{R}$. As before, $\sigma(\lambda)$ is increasing and non-negative.

\subsection{Connecting $\theta_{p}$ and $\sigma(\lambda)$}

We can characterize those functions for which $\theta_{p} \rightarrow \infty$ in terms of $\sigma(\lambda)$.

\section{Theorem 6.1}

Let $f \in S_{\infty}$. Then $\theta_{p} \rightarrow \infty$ if and only if $\sigma(\lambda)<\infty$ for all $\lambda \in \mathbb{R}$. 
Proof. $(\Leftarrow)$ Suppose $\sigma(\lambda)<\infty$ for all $\lambda$. Then for every $\lambda$,

$$
\int_{x}^{2 x}|f|^{p} \geq \int_{U_{\lambda}(x)}|f|^{p} \geq \int_{U_{\lambda}(x)} t^{p \lambda} d t \geq c x^{p \lambda}\left|U_{\lambda}(x)\right|=\Omega\left(x^{p \lambda+1-\sigma(\lambda)-\varepsilon}\right)
$$

for every $\varepsilon>0$. The LHS is $\ll x^{p \theta_{p}+1+\varepsilon}$. Thus

$$
p \theta_{p} \geq p \lambda-\sigma(\lambda) \quad \text { for all } \lambda .
$$

Hence $\theta_{p} \geq \lambda-\frac{\sigma(\lambda)}{p}$ and letting $p \rightarrow \infty$ gives

$$
\liminf _{p \rightarrow \infty} \theta_{p} \geq \lambda .
$$

This is true for every $\lambda$, so $\theta_{p} \rightarrow \infty$ as $p \rightarrow \infty$.

For the converse, suppose $\theta_{p} \rightarrow \infty$. Then, given $\lambda \in \mathbb{R}$, there exists $p$ such that $\theta_{p}>\lambda$. As such

$$
\int_{[x, 2 x] \backslash U_{\lambda}(x)}|f|^{p} \leq c x^{p \lambda+1}=o\left(x^{p \theta_{p}+1-\eta}\right)
$$

for some $\eta>0$. It follows that

$$
\int_{U_{\lambda}(x)}|f|^{p}=\int_{x}^{2 x}|f|^{p}-\int_{[x, 2 x] \backslash U_{\lambda}(x)}|f|^{p}=\Omega\left(x^{p \theta_{p}+1-\varepsilon}\right) \quad \text { for every } \varepsilon>0 .
$$

Now suppose $w(\lambda)=-\infty$. By Cauchy-Schwarz,

$$
\left(\int_{U_{\lambda}(x)}|f|^{p}\right)^{2} \leq \int_{U_{\lambda}(x)} d t \cdot \int_{U_{\lambda}(x)}|f|^{2 p} \leq\left|U_{\lambda}(x)\right| \int_{x}^{2 x}|f|^{2 p} \ll x^{-A}
$$

for all $A>0$, since $\sigma(\lambda)=\infty$. This contradicts (6.2). Hence $\sigma(\lambda)<\infty$ for $\lambda<\theta_{p}$; i.e. $\sigma(\lambda)<\infty$ for all $\lambda$.

\section{Remarks 6.1}

(a) The proof of Theorem 6.1 actually shows that, on writing $\theta_{\infty}=\lim _{p \rightarrow \infty} \theta_{p} \in\left[\theta_{1}, \infty\right]$, we have $\sigma\left(\theta_{\infty}-\right)<\infty$ while $\sigma\left(\theta_{\infty}+\right)=\infty$.

(b) We see from (6.1), with say $p=1$, that $\sigma(\lambda) \geq \lambda-\theta_{1} \rightarrow \infty$ as $\lambda \rightarrow \infty$. In fact, it is even true that $\frac{\sigma(\lambda)}{\lambda} \rightarrow \infty$. For

$$
\liminf _{\lambda \rightarrow \infty} \frac{\sigma(\lambda)}{\lambda} \geq \liminf _{\lambda \rightarrow \infty}\left(p-\frac{p \theta_{p}}{\lambda}\right)=p
$$

for every $p>0$.

Now we show that Theorem 2.2 extends to this setting.

\section{Theorem 6.2}

We have

$$
p \theta_{p}=\sigma^{*}(p)=\sup _{\lambda \in \mathbb{R}}(p \lambda-\sigma(\lambda)) .
$$

Proof. From (6.1) we see already that $p \theta_{p} \geq \sigma^{*}(p)$. It remains to show the reverse inequality.

Fix $\lambda<\theta_{p}$ and let $\mu>\lambda$ (fixed but arbitrary). Let $K \in \mathbb{N}$ (large) and put $\mu_{k}=\lambda+\frac{k}{K}(\mu-\lambda)$ for $k=0,1, \ldots, K$. Thus $\lambda=\mu_{0}<\ldots<\mu_{K}=\mu$. As such

$$
\int_{U_{\lambda}(x)}|f|^{p}=\int_{U_{\mu}(x)}|f|^{p}+\sum_{k=1}^{K} \int_{U_{\mu_{k-1}}(x) \backslash U_{\mu_{k}}(x)}|f|^{p} .
$$


For $t \in U_{\mu_{k-1}}(x) \backslash U_{\mu_{k}}(x)$, we have $|f(t)| \leq t^{\mu_{k}} \leq c x^{\mu_{k}}$. Hence

$$
\int_{U_{\mu_{k-1}}(x) \backslash U_{\mu_{k}}(x)}|f|^{p} \leq c x^{p \mu_{k}}\left|U_{\mu_{k-1}}(x)\right| \ll x^{p \mu_{k}+1-\sigma\left(\mu_{k-1}\right)+\varepsilon} \leq x^{\sigma^{*}(p)+1+\frac{p(\mu-\lambda)}{K}+\varepsilon} .
$$

Hence the RHS sum of (6.3) is $O\left(x^{\sigma^{*}(p)+1+\frac{p(\mu-\lambda)}{K}+\varepsilon}\right)$. Suppose for a contradiction that $p \theta_{p}>\sigma^{*}(p)$ for some $p$. Then we can choose $K$ so large that $\sigma^{*}(p)+\frac{p(\mu-\lambda)}{K}<p \theta_{p}$. As such, the RHS sum in (6.3) is $o\left(x^{p \theta_{p}+1-\eta}\right)$ for some $\eta>0$ and so, for some $c>0$,

$$
\int_{U_{\mu}(x)}|f|^{p} \geq \int_{U_{\lambda}(x)}|f|^{p}-c x^{p \theta_{p}+1-\eta}=\Omega\left(x^{p \theta_{p}+1-\varepsilon}\right) \quad \text { for all } \varepsilon>0 \text { for every } \mu .
$$

But, using Cauchy-Schwarz again,

$$
\int_{U_{\mu}(x)}|f|^{p} \leq \sqrt{\left|U_{\mu}(x)\right| \int_{x}^{2 x}|f|^{2 p}} \ll x^{\frac{1}{2}-\frac{1}{2} \sigma(\mu)+\frac{1}{2}+p \theta_{2 p}+\varepsilon},
$$

so (6.4) implies $\sigma(\mu) \leq 2 p\left(\theta_{2 p}-\theta_{p}\right)$. This is true for every $\mu>\lambda$ as $\mu$ was chosen arbitrarily. Thus $\sigma(\cdot)$ is bounded above. This contradicts the fact that $\sigma(\mu) \geq p \mu-p \theta_{p} \rightarrow \infty$ as $\mu \rightarrow \infty$.

As before we have $\sigma(\lambda) \geq p \lambda-p \theta_{p}$, so

$$
\sigma(\lambda) \geq \sup _{p>0} p \lambda-p \theta_{p}=\tilde{\sigma}(\lambda) .
$$

Proposition 2.8(b) also generalizes to this setting. As $\theta_{p}$ is increasing, $\theta_{0}=\lim _{p \rightarrow 0+} \theta_{p}$ exists in $\mathbb{R} \cup\{-\infty\}$.

\section{Proposition 6.3}

Suppose $\theta_{0}>-\infty$. Then $\sigma(\lambda)=0$ for $\lambda<\theta_{0}$ and $\sigma(\lambda)>0$ for $\lambda>\theta_{0}$.

Proof. First observe that for $\lambda<\theta_{0}$ and $p>0, p \lambda-\sigma(\lambda) \leq p \lambda<p \theta_{p}$. Thus

$$
p \theta_{p}=\sup _{\lambda \geq \theta_{0}-}(p \lambda-\sigma(\lambda)) .
$$

After Remark 6.1, there exists $\lambda_{0}>0$ such that $\sigma(\lambda) \geq \lambda$ for $\lambda \geq \lambda_{0}$. As such, $p \lambda-\sigma(\lambda) \leq 0$ for $p \in(0,1]$. But $p \theta_{p} \rightarrow 0$ as $p \rightarrow 0+$, so by continuity, $p \_p>0$ for $p$ sufficiently small. Thus for such $p$,

$$
p \theta_{p}=\sup _{\theta_{0}-\leq \lambda \leq \lambda_{0}}(p \lambda-\sigma(\lambda)) \leq p \lambda_{0}-\sigma\left(\theta_{0}-\right) .
$$

Letting $p \rightarrow 0+$, gives $\sigma\left(\theta_{0}-\right) \leq 0$. Hence $\sigma\left(\theta_{0}-\right)=0$ as required.

For the second part, if $\lambda>\theta_{0}$, then $\exists p>0$ such that $\lambda>\theta_{p}$. As such, $\sigma(\lambda) \geq p\left(\lambda-\theta_{p}\right)>0$.

\section{Theorem 6.4}

Let $k:[\alpha, \infty) \rightarrow \mathbb{R}$ denote an arbitrary continuous and stricly increasing function for which $k(\alpha)=0$ and $k(\lambda) / \lambda \rightarrow \infty$ as $\lambda \rightarrow \infty$. Then there exists $f \in S_{\infty}$ such that

$$
\sigma(\lambda)=\left\{\begin{array}{cc}
k(\lambda) & \text { for } \lambda \geq \alpha \\
0 & \text { for } \lambda<\alpha
\end{array}\right.
$$

Proof Let $f(t)=t^{\tau(t)}$ for $t \geq 1$ where $\tau(t)$ is defined on intervals $\left[2^{n}, 2^{n+1}\right]\left(n \in \mathbb{N}_{0}\right)$ as follows:

$$
\tau\left(\frac{3}{2} 2^{n} \pm \frac{1}{2} 2^{n(1-k(\lambda))}\right)= \begin{cases}\lambda & \text { if } \alpha \leq \lambda \leq \lambda_{n} \\ \lambda_{n} & \text { if } \lambda>\lambda_{n}\end{cases}
$$

Here $\lambda_{n}$ is any strictly increasing sequence tending to infinity such that $\lambda_{n} \geq \alpha$ and $k\left(\lambda_{n}\right) \ll 2^{b n}$ for some $b$. As such, $\tau\left(2^{n}\right)=\alpha$, and, since $\lambda_{n} \rightarrow \infty, \theta=\infty$. 
Now given $\lambda \in\left[\alpha, \lambda_{n}\right]$ and $t \in\left[2^{n}, 2^{n+1}\right]$, we have $f(t) \geq t^{\lambda}$ if and only if $\tau(t) \geq \lambda$. Thus

$$
U_{\lambda}\left(2^{n}\right)=\left[\frac{3}{2} 2^{n}-\frac{1}{2} 2^{n(1-k(\lambda))}, \frac{3}{2} 2^{n}+\frac{1}{2} 2^{n(1-k(\lambda))}\right]
$$

and so $\left|U_{\lambda}\left(2^{n}\right)\right|=2^{n(1-k(\lambda))}$. Thus, taking $x \in\left[2^{n}, 2^{n+1}\right]$,

$$
\left|U_{\lambda}(x)\right| \leq\left|U_{\lambda}\left(2^{n}\right)\right|+\left|U_{\lambda}\left(2^{n+1}\right)\right| \leq\left(1+2^{1-k(\lambda)}\right) 2^{n(1-k(\lambda))} \ll x^{1-k(\lambda)},
$$

and so $\sigma(\lambda)=k(\lambda)$ for $\lambda \geq \alpha$. Since $k(\alpha)=0$ and $\sigma$ is increasing, it follows that $\sigma(\lambda)=0$ for $\lambda<\alpha$.

Now it remains to show that $\theta_{p}$ exists for this $f$. It suffices to show that $\int_{x}^{2 x}|f|^{p} \ll x^{A}$ for some $A$ (depending on $p$ ) and moreover it is enough to consider $x=2^{n}$. As such, we have

$$
\begin{aligned}
\int_{2^{n}}^{2^{n+1}}|f|^{p} & \asymp \int_{2^{n}}^{2^{n+1}} 2^{n p \tau(t)} d t=2 \int_{0}^{2^{n-1}} 2^{n p \tau\left(\frac{3}{2} 2^{n}+y\right)} d y \\
& =2^{n\left(p \lambda_{n}+1-k\left(\lambda_{n}\right)\right)}+n \log 2 \int_{\alpha}^{\lambda_{n}} 2^{n(p \lambda+1-k(\lambda))} d k(\lambda) .
\end{aligned}
$$

But $p \lambda+1-k(\lambda) \rightarrow-\infty$ as $\lambda \rightarrow \infty$, so the first term tends to zero while the integrand is bounded by $2^{a n}$ with $a=\sup _{\lambda \geq \alpha}(p \lambda+1-k(\lambda))$. Hence

$$
\int_{2^{n}}^{2^{n+1}}|f|^{p} \ll n 2^{a n} \int_{\alpha}^{\lambda_{n}} d k(\lambda) \ll n 2^{a n} k\left(\lambda_{n}\right) \ll 2^{c n},
$$

for some $c$.

\section{Theorem 6.5}

Let $\phi_{p} \in \mathbb{R}(p>0)$ be increasing without bound such that $p \phi_{p}$ is convex. Suppose further that $\phi_{p} \rightarrow \phi_{0}>$ $-\infty$ as $p \rightarrow 0+$. Then there exists $f \in S_{\infty}$ such that $\theta_{p}=\phi_{p}$.

Proof. As in the proof of Theorem 2.5, let $g(\lambda)=\sup _{p>0}\left(p \lambda-p \phi_{p}\right)$, which is convex and increasing. By taking arbitrarily close to 0 and using the condition $\lim _{p \rightarrow 0+} \phi_{p}>-\infty$ implies that $g \geq 0$. Further, as in Proposition 2.8, $g(\lambda)=0$ for $\lambda<\phi_{0}$ while $g(\lambda)>0$ for $\lambda>\phi_{0}$. By convexity, $g$ is therefore strictly increasing om $\left[\phi_{0}, \infty\right)$. By Theorem 6.4, there exists $f \in S_{\infty}$ with $\sigma(\lambda)=g(\lambda)$ for all $\lambda$. Hence

$$
p \phi_{p}=\sup _{\lambda}(p \lambda-g(\lambda))=\sup _{\lambda}(p \lambda-\sigma(\lambda))=p \theta_{p}
$$

as required.

\subsection{A special class of arithmetical functions}

Now we consider $f$ of the form

$$
f(x)=F\left(\left[e^{x-1}\right]\right),
$$

where $F$ is an arithmetical function. In fact we shall restrict further to non-negative multiplicative functions which are $O\left(n^{\varepsilon}\right)$. As such the size of $\theta_{p}$ is largely determined by the behaviour of $F$ at the primes. To ensure $\theta=\infty$, we need $F(n)=\Omega\left((\log n)^{A}\right)$ for all $A$.

\section{Theorem 6.6}

Let $f$ be of the form (6.6) where $F$ is multiplicative and non-negative. Further assume that $F(n) \ll n^{\varepsilon}$ for all $\varepsilon>0$ and $F(p) \rightarrow \alpha>0$ as $p \rightarrow \infty$ through primes. Then $f \in S_{\infty}$ with $\theta_{q}=\frac{\alpha^{q}-1}{q}{ }^{7}$

Proof. Let $g(n)=\frac{F(n)^{q}}{n}$, which is multiplicative and non-negative. Further $g(n) \ll n^{-1+\varepsilon}$ for all $\varepsilon>0$ and

$$
\sum_{e^{x}<p \leq e^{\lambda x}} g(p)=\sum_{e^{x}<p \leq e^{\lambda x}} \frac{F(p)^{q}}{p} \sim \alpha^{q} \log \lambda
$$

\footnotetext{
${ }^{7}$ We use $q$ here, as primes will be denoted by $p$.
} 
as $x \rightarrow \infty$ for every $\lambda>0$. Thus $g$ satisfies the conditions of Theorem 6.3 .2 of [3]; namely,

$$
\sum_{p, k \geq 2} g\left(p^{k}\right), \sum_{p} g(p)^{2}<\infty
$$

and

$$
\sum_{x<\log p \leq \lambda x} g(p) \rightarrow b \log \lambda
$$

as $x \rightarrow \infty$ for every $\lambda>0$ for some $b \geq 0$. As such, Theorem 6.3.2. says that $\sum_{n \leq e^{x}} g(n)$ is regularlyvarying of index $\alpha^{q}$; in particular this means

$$
\sum_{n \leq e^{x}} \frac{F(n)^{q}}{n}=x^{\alpha^{q}+o(1)}
$$

But, on writing $F(x)=F([x])$,

$$
\begin{aligned}
\int_{0}^{x}|f|^{q} & =\int_{1}^{x} F\left(e^{t-1}\right)^{q} d t=\int_{1}^{e^{x-1}} \frac{F(y)^{q}}{y} d y \\
& =\sum_{n \leq e^{x-1}} \int_{n}^{n+1} \frac{F(y)^{q}}{y} d y+\int_{\left[e^{x-1}\right]}^{e^{x-1}} \frac{F(y)^{q}}{y} d y \\
& =\sum_{n \leq e^{x-1}} F(n)^{q} \log \left(1+\frac{1}{n}\right)+O\left(\frac{F\left(e^{x-1}\right)^{q}}{e^{x-1}}\right) .
\end{aligned}
$$

The $O$-term is $o(1)$ since $F(x) \ll x^{\varepsilon}$ and the first sum is $x^{\alpha^{q}+o(1)}$ by (5.6). Hence the result follows.

Remarks 6.2 (i) Note that $\theta_{p} \rightarrow 0$ as $p \rightarrow \infty$ if $\alpha \leq 1$.

(ii) Using (6.5) we have, in case $\alpha>1$,

$$
\tilde{\sigma}(\lambda \log \alpha)=\left\{\begin{array}{cc}
\lambda \log \lambda-\lambda+1 & \text { if } \lambda>1 \\
0 & \text { if } \lambda \leq 1
\end{array},\right.
$$

while if $\alpha \leq 1$

$$
\tilde{\sigma}(\lambda)=\left\{\begin{array}{cc}
\infty & \text { if } \lambda>0 \\
0 & \text { if } \lambda \leq 0
\end{array}\right.
$$

(iii) The result easily generalises to the case where $F(p)$ oscillates asymptotically between different values along different arithmetic progressions. If $f$ satisfies the conditions of Theorem 6.6, but now $F(p) \rightarrow \alpha_{k}$ as $p \rightarrow \infty$ through primes $p \equiv k(\bmod m)$, with $k \leq m$ coprime to $m$, then $f \in S_{\infty}$ with

$$
q \theta_{q}=\frac{1}{\varphi(m)} \sum_{\substack{k \leq m \\(k, m)=1}}\left(\alpha_{k}^{q}-1\right)
$$

For this time

$$
\sum_{e^{x}<p \leq e^{\lambda x}} \frac{F(p)^{q}}{p} \sim \sum_{\substack{k \leq m \\(k, m)=1}} \alpha_{k}^{q} \sum_{\substack{e^{x}<p \leq e^{\lambda x} \\ p \equiv k(\bmod m)}} \frac{1}{p} \rightarrow \frac{1}{\varphi(m)}\left(\sum_{\substack{k \leq m \\(k, m)=1}} \alpha_{k}^{q}\right) \log \lambda
$$

as $x \rightarrow \infty$. Again, the conditions of Theorem 6.3.2 of [3] are satisfied and the result follows.

\subsection{Examples}

(a) Take $f(x)=d\left(\left[e^{x-1}\right]\right)$ where $d(n)$ is the divisor function. By Theorem $6.6, f \in S_{\infty}$ with $\theta_{p}=\frac{2^{p}-1}{p}$. Hence $\theta_{0}=\log 2$ and by Theorem 6.3, $\sigma(\lambda)=0$ for $\lambda<\log 2$ and positive for $\lambda>\log 2$. Indeed $\sigma(\lambda \log 2) \geq \tilde{\sigma}(\lambda \log 2)=\int_{1}^{\lambda} \log t d t$ for $\lambda>1$. 
(b) Take $f(x)=F\left(\left[e^{x-1}\right]\right)$ where $F(n)=\sum_{d \mid n} \chi(d)$, where $\chi(n)=(-1)^{\frac{n-1}{2}}$ for $n$ odd and zero otherwise. Thus $F(n)=\frac{1}{4} r(n)$, where $r(n)$ is the number of ways of writing $n$ as a sum of two squares. In this case, for $p$ an odd prime

$$
F(p)= \begin{cases}2 & \text { if } p \equiv 1(\bmod 4) \\ 0 & \text { if } p \equiv 3(\bmod 4)\end{cases}
$$

By Remarks 6.2(iii), $f \in S_{\infty}$ we have

$$
\theta_{p}=\frac{2^{p-1}-1}{p}
$$

Note that here $\theta_{0}=-\infty$ and $\sigma(\lambda)>0$ for every $\lambda$.

Indeed, (6.5) shows after a little calculation that

$$
\tilde{\sigma}(\lambda \log 2)=\left\{\begin{array}{cl}
\lambda \log 2 \lambda-\lambda+1 & \text { if } \lambda>\frac{1}{2} \\
\frac{1}{2} & \text { if } \lambda \leq \frac{1}{2}
\end{array} .\right.
$$

(c) Let $f(x)=\left|\zeta\left(\frac{1}{2}+i e^{x}\right)\right|^{2}$ for $x \geq 1$ and zero otherwise. On the Riemann Hypothesis, it was shown that

$$
T(\log T)^{p^{2}} \ll I_{p}(T)=\int_{0}^{T}\left|\zeta\left(\frac{1}{2}+i t\right)\right|^{2 p} d t \ll T(\log T)^{p^{2}+\varepsilon}
$$

for every $p>0$ and $\varepsilon>0$. The lower bound (which holds unconditionally for $p$ rational) is by Ramachandra [22] and Heath-Brown [10], while the upper bound is due to Soundararajan [27], which has recently been improved to $\varepsilon=0$ by Harper [9] (all on RH). Hence

$$
\int_{x}^{2 x}|f|^{p}=\int_{x}^{2 x}\left|\zeta\left(\frac{1}{2}+i e^{y}\right)\right|^{2 p} d y=\left[\frac{I_{p}(t)}{t}\right]_{e^{x}}^{e^{2 x}}+\int_{e^{x}}^{e^{2 x}} \frac{I_{p}(t)}{t^{2}} d t
$$

Assuming $\mathrm{RH}$, the first term is $\ll x^{p^{2}+\varepsilon}$ while the integral is $x^{p^{2}+1+o(1)}$. Thus $f \in S_{\infty}$ with $\theta_{p}=p$. Now $\theta_{0}=0$, so by Theorem $6.3, \sigma(\lambda)=0$ for $\lambda<0$. For $\lambda \geq 0,(6.5)$ gives $\tilde{\sigma}(\lambda)=\frac{\lambda^{2}}{4}$. Thus, on $\mathrm{RH}$, we find that for every $a \geq 0$ and every $\varepsilon>0$,

$$
\left|\left\{t \in[x, 2 x]:\left|\zeta\left(\frac{1}{2}+i e^{t}\right)\right| \geq t^{a}\right\}\right| \ll x^{1-a^{2}+\varepsilon} .
$$

(d) Let $f(x)=\left|S\left(e^{e^{x}}\right)\right|$, where $S(t)=\frac{1}{\pi} \arg \zeta\left(\frac{1}{2}+i t\right)$. Selberg [24] showed that $\int_{0}^{T} S(t)^{2 k} d t \sim$ $\frac{(2 k) !}{k !(2 \pi)^{2 k}} T\left((\log \log T)^{k}\right.$ for each positive integer $k$. This easily leads to $\int_{x}^{2 x}|f|^{2 k} \sim a_{k} x^{k+1}$ for some constant $a_{k}>0$. Thus $\theta_{2 k}=\frac{1}{2}$ for $k \in \mathbb{N}$ while $\theta=\infty$ (since $S(t)$ is sometimes as large as a power of $\log t$ ). It follows that $\theta_{p}=\frac{1}{2}$ for all $p>0$. Thus also $\sigma(\lambda)=\tilde{\sigma}(\lambda)=\infty$ for $\lambda>\frac{1}{2}$ and zero otherwise.

\section{References}

[1] T. M. Apostol, Introduction to Analytic Number Theory, Springer-Verlag, 1976.

[2] W. Beckner, Inequalities in Fourier Analysis, Annals of Math. 102 (1975) 159-182.

[3] N. H. Bingham, C. M. Goldie, and J. L. Teugels, Regular variation, Cambridge University Press, 1987.

[4] J. Bourgain, Decoupling, exponential sums and the Riemann zeta-function, preprint available at arXiv.1408.5794.

[5] H. Cramér, Űber zwei Såtze von Herrn G. H. Hardy, Math. Zeit. 15 (1922) 200-210.

[6] P. Erdős, On a family of symmetric Bernoulli convolutions, Amer. J. Math. 61 (1939) 974âĂŞ976.

[7] G. H. Hardy, A. E. Ingham and G. Pólya, Theorems concerning mean values of analytic functions, Proc. Royal Society (A) 113 (1927) 542-569. 
[8] G. H. Hardy and E. M. Wright, An introduction to the theory of numbers, (fifth edition) Oxford University Press, 1979.

[9] A. J. Harper, Sharp conditional bounds for moments of the Riemann zeta function, preprint (2013), http://arxiv.org/abs/1305.4618

[10] D. R. Heath-Brown, Fractional moments of the Riemann Zeta-function, Jour. LMS 24 (1981) 65-78.

[11] D. R. Heath-Brown, The Dirichlet divisor problem, Advances in Number Theory, OUP (New York), 1993.

[12] T. W. Hilberdink, Some connections between Bernoulli convolutions and Analytic Number Theory, Proceedings of Symposia in Pure Mathematics 72 (2004) 233-271.

[13] M. N. Huxley, Exponential sums and lattice points III, Proc. LMS 87 (2003) 591-609.

[14] M. N. Huxley, Exponential sums and the Riemann zeta function V, Proc. LMS 90 (2005) 1-41.

[15] A. Ivić, The Riemann Zeta-Function; theory and applications, Dover Publications, 2003.

[16] A. Ivić, The Mellin transform of powers of the Riemann zeta function, Acta Arithmetica 95 (2000) 305-342.

[17] A. Ivić, The Laplace and Mellin transforms of powers of the Riemann zeta function, Int. J. Math. Anal. 1 (2006) 113-140.

[18] M. Jutila, the Mellin transform of the square of Riemann's zeta function, periodica Math. Hung. 42 (2001) 179-190.

[19] J.-P. Kahane, The last problem of Harald Bohr, J. Austral. Math. Soc. 47 (1989) 133-152.

[20] J. P. Kahane and R. Salem, Sur la convolution d'une infinité de distributions de Bernoulli, Colloq. Math. 6 (1958), 193-202.

[21] C. Niculescu and L.-E. Persson, Convex functions and their applications, CMS books in Mathematics, Springer, 2005.

[22] K. Ramachandra, Some remarks on the mean value of the Riemann zeta-function and other Dirichlet series I, Hardy-Ramanujan Journal 1 (1978) 1-15.

[23] K. Ramachandra, On the zeros of a class of generalised Dirichlet series V, Journal Reine und Angew. Math. 303/304 (1978) 295-313.

[24] A. Selberg, Contributions to the theory of the Riemann zeta function, Arch. for Math. og Naturv. B, 48 (1946), no.5.

[25] P. Shmerkin, On the exceptional set for absolute continuity of Bernoulli convolutions, Geom. Funct. Anal. 24 (2014) 946-958.

[26] B. Solomyak, On the random series $\sum \pm \lambda^{n}$ (an Erdős problem), Ann. of Math. 142 (1995) 611âĂŞ625.

[27] K. Soundararajan, Moments of the Riemann zeta function, Annals of Mathematics, 170 (2009) $981-993$.

[28] E. C. Titchmarsh, The Theory of Functions, Second edition, Oxford University Press, 1986.

[29] E. C. Titchmarsh, The Theory of the Riemann Zeta-function, Second edition, Oxford University Press, 1986.

[30] E. C. Titchmarsh, Introduction to the Theory of Fourier integrals, Oxford University Press, 1937.

[31] D. V. Widder, The Laplace Transform, Princeton University Press, 1946. 